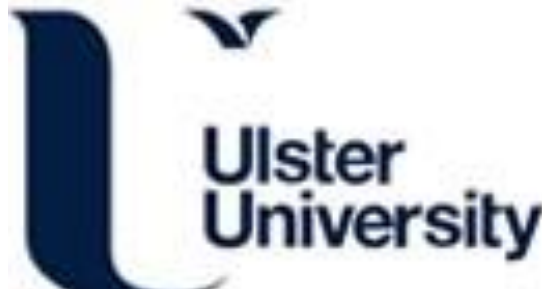

\section{Computation of CNN's Sensitivity to Input Perturbation}

Xiang, L., Zeng, X., Wu, S., Liu, Y., \& Yuan, B. (Accepted/In press). Computation of CNN's Sensitivity to Input Perturbation. Neural Processing Letters.

Link to publication record in Ulster University Research Portal

Published in:

Neural Processing Letters

Publication Status:

Accepted/In press: 23/12/2020

\section{Document Version}

Author Accepted version

\section{General rights}

Copyright for the publications made accessible via Ulster University's Research Portal is retained by the author(s) and / or other copyright owners and it is a condition of accessing these publications that users recognise and abide by the legal requirements associated with these rights.

\section{Take down policy}

The Research Portal is Ulster University's institutional repository that provides access to Ulster's research outputs. Every effort has been made to ensure that content in the Research Portal does not infringe any person's rights, or applicable UK laws. If you discover content in the Research Portal that you believe breaches copyright or violates any law, please contact pure-support@ulster.ac.uk. 


\title{
Computation of CNN's Sensitivity to Input Perturbation
}

\author{
Lin Xiang ${ }^{1,2}$, Xiaoqin Zeng ${ }^{1, *}$, Shengli $\mathrm{Wu}^{3}$, Yanjun $\mathrm{Liu}^{1}$, Baohua Yuan ${ }^{1}$
}

\begin{abstract}
Although Convolutional Neural Networks (CNNs) are considered as being "approximately invariant" to nuisance perturbations such as image transformation, shift, scaling, and other small deformations, some existing studies show that intense noises can cause noticeable variation to CNNs' outputs. This paper focuses on exploring a method of measuring sensitivity by observing corresponding output variation to input perturbation on CNNs. The sensitivity is statistically defined in a bottom-up way from neuron to layer, and finally to the entire CNN network. An iterative algorithm is proposed for approximating the defined sensitivity. On the basic architecture of CNNs, the theoretically computed sensitivity is verified on the MNIST database with four types of commonly used noise distributions: Gaussian, Uniform, Salt \& Pepper, and Rayleigh. Experimental results show the theoretical sensitivity is on the one hand in agreement with the actual output variation what on the maps, layers or entire networks are, and on the other hand an applicable quantitative measure for robust network selection.
\end{abstract}

Keywords Convolutional Neural Network, Sensitivity, Additive Noise, Input Perturbation

\section{Introduction}

Convolutional neural networks (CNNs) have been extensively studied in the last few years and achieved very good performance for various tasks, especially computer vision [1-9]. Due to the capability of joint feature and classifier learning, CNNs are deemed to be invariant to small image distortion [10,11]. However, latest researches indicate that CNNs are vulnerable to input perturbations such as randomly added noise, adversarial samples, and even agnostic perturbations. Slight input perturbation, sometimes imperceptible to human eyes can lead to dramatic change in CNNs' performance and completely fool the network [12]. Such vulnerability of CNNs has cast doubt on its ability and reliability in safety-critical applications, such as self-driving or face recognition. It has started raising an interesting question about how trustworthy CNNs are and stimulated a wide range of research on the effect of input noise to CNNs' output.

Both empirical and theoretical works have emerged to study the influence of various forms of input perturbations [13-25]. In most research works, different measures of robustness are used to quantify the variation of CNNs' output to external perturbations that affect input samples. The discrete classification function to input perturbation is the most common criterion [26]. It is worth noting that most of these measures, e.g. robustness, reflects output variation of a network to input

\footnotetext{
* Corresponding author. E-mail: xzeng@hhu.edu.cn

${ }^{1}$ Institute of Intelligence Science and Technology, Hohai University, Nanjing 210098, China

${ }^{2}$ Huaiyin Institute of Technology, Huai' an 223003, China

${ }^{3}$ School of Computing, Ulster University, Belfast UK
} 
perturbations by means of the changes of other indexes, such as accuracy. In contrast, this work seeks an effective way to directly quantify the response of CNNs to input perturbations by a thorough study of the relationship between inputs and outputs.

Sensitivity analysis is one of the main tools used in exploring the uncertainty of models. For a generic black-box representation of a model $y=f(x)$, in which $x=\left[x_{1}, x_{2}, \ldots, x_{n}\right]$ is an input vector and $y$ is a scalar output, sensitivity analysis is to study how uncertainty in the output of the model can be apportioned among different sources of uncertainty in the model's input [27,28]. Local and global methods are the two main approaches of sensitivity analysis [29]. Local methods define sensitivity as a partial derivative of the model's output with respect to one of its inputs. Partial derivative, $\partial y / \partial x_{i}$, is simple and effective for linear models. On the other hand, global methods address this problem by considering the set of all possible inputs in a hypercube space, which are more fit for nonlinear features and interactions between features in the model [30]. Sensitivity analysis on neural networks can be traced back to the 1960s. Initially it was used to investigate the probability of misclassification due to weight perturbations, noisy input and machine imprecision. With the in-depth study, sensitivity analysis on neural networks has been extensively applied to network structure optimization, weights pruning and model selection. A variety of studies on sensitivity analysis have emerged for different types of neural networks. [31-34] focus on sensitivity of simple discrete networks of Adalines and Madalines, while more works dwell on continuous neural networks, like the most common MLPs [35-40], RBFs [41], Neocognitrons [42] and other continuous networks [43-49]. As far as the research methods to be concerned, there are two different categories: geometrical and statistical approaches.

Sensitivity analysis of CNNs is the major objective of this paper. The most relevant work was done by Zhang in [50]. That research is an empirical study. It positions a simple one-layer CNN as a new standard baseline model akin to bag-of-words SVMs and logistic regression. An extensive experiment is conducted to analyze CNNs for sentence classification, distinguishing between important and comparatively inconsequential design decisions. Although based on similar CNN models, this paper attempts to quantify sensitivity to input perturbations through theoretical calculation, rather than empirical investigation as in [50]. Global sensitivity analysis is taken into consideration for the nonlinearity of CNNs. Moreover, for the distinctive characters of weight sharing and local connection, sensitivity analysis for CNNs is offered in this paper in a different way from that of discrete or continuous fully-connected networks.

The overall goal of this paper is to provide a quantitative method for measuring the effect of CNNs to input perturbations by sensitivity analysis, instead of robustness or other alternatives. For a deterministic CNN, we focus on the sensitivity due to input perturbations, without considering inner weight perturbations. Then the assessment of uncertainty existing in output boils down to uncertainty propagation from input to output. Therefore, a bottom-up approach from neuron, layer, and network is proposed to consider the propagation of the sensitivity layer by layer. Statistical variation of the output to all possible input perturbations is defined as sensitivity of the model and a numerical calculation algorithm is proposed to estimate the defined sensitivity with the normal distribution assumption. The main contributions of this paper are as follows:

(1) Virtual neurons are introduced to be the basic component of CNNs, based on which the architecture of the CNN can be equally represented in a fully-connected instead of locally-connected way; 
(2) We define sensitivity of CNNs with the fully-connected architecture in a bottom-up style from virtual neurons to layers to the entire network;

(3) We propose an iterative algorithm CNN_SEN_INP to compute the defined sensitivity;

(4) The Chi-square distance is used to measure the difference between the calculated sensitivity and the simulation results.

The rest of this paper is organized as follows. Section 2 introduces the model of CNN and notations for defining sensitivity. The sensitivity definitions for a single neuron, a layer and an entire CNN are successively given in Section 3. Section 4 discusses the computation of sensitivity and proposes an algorithm for that. Empirical verification of the proposed algorithm and corresponding discussions are given in Section 5. Finally, the conclusion is drawn in Section 6.

\section{CNN model and notations}

As a multi-layer feedforward neural network, a CNN is stacked by convolutional, pooling, and fully-connected layers. Each convolutional or pooling layer is consisted of multiple 2-D feature maps, and each map contains multiple independent neurons [51]. 2-D or 3-D visual patterns are directly input to the network, simplifying the knowledge representation of data. In the convolutional layers, also known as feature extraction layers, features are extracted by convolution kernels to constitute the feature maps of the layers. Because different kernels extract different local features, multiple kernels are set to extract different local features on the convolutional layers. By aggregating different features, the pooling operation retains the most significant features and reduces the dimension of the feature maps. To some extent, pooling can be regarded as a convolution operation with fixed parameters, such as mean pooling. For the sake of simplicity, the convolutional layers and pooling layers are collectively referred to as the convolutional layers in the rest of this paper, without any further distinction. After multiple alternately interconnected convolution layers and pooling layers, the significant features are sent through fully-connected layers to accomplish the task of classification or recognition. In the whole process of pattern recognition, the convolutional layers for extracting features are the core of CNNs, and it is also one of the important characteristics different from other neural networks. So we introduce the mathematical representation of convolution operation in detail, and then give the equivalent fully-connected structure of CNNs.

Suppose there is a given CNN with $L$ layers, $L_{C}$ convolution and pooling layers and $L_{f}$ fullyconnected layers, $L=L_{c}+L_{f}$. For simplicity without losing generality, the sliding step of kernels in the convolution layer is set to 1 without padding, the average pooling is chosen with the kernel size of $2 \times 2$ and the input and convolution kernels are both square matrices. Tensors are used to represent inputs, outputs, kernels and feature maps in convolutional layers.

Because the input of layer $l$ is just the output of layer $l-1$, a 3rd-order tensor $\boldsymbol{X}^{l-1} \in \boldsymbol{R}^{H^{l-1} \times W^{l-1} \times C^{l-1}}$ is used to represent the input of the convolution layer $l$, among which $C^{l-1}$ is the number of feature maps in layer $l-1, H^{l-1}$ and $W^{l-1}$ are the size of each map. Especially $\boldsymbol{X}^{\mathbf{0}} \in \boldsymbol{R}^{H \times W \times C}$ denotes an input image, where $C$ is the number of channels ( $C=1$ for gray, $C=3$ for RGB images), $H$ and $W$ are the size of the image in each channel. Similarly $\boldsymbol{X}^{l} \in \boldsymbol{R}^{H^{l} \times W^{l} \times C^{l}}$ is denoted as the output of layer $l$, and we have:

$$
\boldsymbol{X}^{\boldsymbol{l}-\mathbf{1}} \underset{\otimes}{\stackrel{\text { Convolution }}{\longrightarrow}} \boldsymbol{V}^{\boldsymbol{l}-\mathbf{1}} \stackrel{\text { Transfer }}{\longrightarrow} \boldsymbol{X}^{\boldsymbol{l}}
$$

where $V^{l-1}$ is the convolved result of input $X^{l-1}, \otimes$ denotes the operation of convolution, and $f$ is the transfer function. 
In each of the convolutional layers, kernels are locally connected to the input. The locally connected region of the input is called a local receptive field. The larger the size of the local receptive field is, the larger the range of original images it can touch. It indicates that more global and semantic features can be contained. On the contrary, the smaller the size, the more local and detailed features it captures. Sharing the same weights in the unit of the local receptive field, the kernel slides over the input map to accomplish the convolution. Let the kernel size be $k \times k$, and $S^{l}$ local receptive fields are generated. Then the input can be represented as a matrix by local receptive fields:

$$
X^{l-1}=\left(x_{i, j}^{l-1}\right)_{C^{l-1} \times S^{l}}
$$

where $\boldsymbol{x}_{i, j}^{l-1}\left(i=1,2, \cdots, C^{l-1}, i=1,2, \cdots, S^{l}\right)$ is the $j^{\text {th }}$ local receptive field in the $i^{\text {th }}$ map.

Let a 4th-order tensor $\boldsymbol{K}^{l} \in \boldsymbol{R}^{C^{l} \times C^{-1} \times k \times k}$ be the kernel bank in layer $l$. Here $C^{l}$ and $C^{l-1}$ are the numbers of the feature maps of layer $l$ and $l-1$, which correspond to the number of kernels in the bank and the number of channels of each kernel respectively. $k$ is the size of one channel of each kernel in the bank, assuming that all the kernels are equal in size.

For a neuron $j$ on map $m$ at layer $l$, the result after convolution is denoted as $v_{m, j}^{l}$ :

$$
v_{m, j}^{l}=\sum_{i=1}^{c^{l-1}} \sum \boldsymbol{k}_{m, i}^{l} \circ \boldsymbol{x}_{i, j}^{l-1}
$$

in which ${ }^{\circ}$ is the Hadamard product, a multiplication with corresponding elements in the matrices, $\boldsymbol{k}_{m, i}^{l}$ is the convolution kernel and $\boldsymbol{x}_{i, j}^{l-1}$ is the local receptive field connected to the kernel. The number of kernels should be the same as the number of input feature maps and equal to $C^{l-1}$. The sum of the Hadamard product of the kernel with local receptive field is convolution $\otimes$, then the above formula is abbreviated as:

$$
v_{m, j}^{l}=\boldsymbol{k}_{m}^{l} \otimes \boldsymbol{x}_{j}^{l-1}
$$

After adding the bias $b_{m, j}^{l}$ and sending to the transfer function $f$, the output $x_{m, j}^{l}$ is obtained:

$$
x_{m, j}^{l}=f\left(\boldsymbol{k}_{m}^{l} \otimes \boldsymbol{x}_{j}^{l-1}+b_{m, j}^{l}\right)
$$

Convolution kernels slides over the input feature maps in the unit of the local receptive field. In the simplest way, it is assumed that the sliding step is only 1 at a time. For a kernel with the size of $k \times k$ and a map $H^{l-1} \times W^{l-1}$, the number of generated local receptive fields is:

$$
S^{l}=H^{l} \times W^{l}
$$

Due to the edge effect, $H^{l}=H^{l-1}-k+1, \quad W^{l}=W^{l-1}-k+1$. Correspondingly, $S^{l}$ outputs are generated to form an output feature map. Regarding the $S^{l}$ output values originated from the same kernel as the output components of a virtual neuron, the correspondence between the output feature map and the neuron is formed.

Supposing that there is a virtual neuron $\mathrm{CNNr}_{m}\left(m=1,2, \cdots, C^{l}\right), S^{l}$ generated local receptive fields are taken as inputs, which are fully connected to $\mathrm{CNNr}_{m}$ and share the same weight matrix (that is, the same kernel) to generate $S^{l}$ outputs after the convolution and transfer function to constitute the neuron's output $\boldsymbol{X}_{m}^{l}$ (i.e. the $m^{\text {th }}$ output feature map). Fig. 1 shows the input-output structure of the virtual neuron. The output of $\mathrm{CNNr}_{m}$ can be expressed as:

$$
\boldsymbol{X}_{m}^{l}=f\left(\boldsymbol{K}_{m}^{l} \otimes \boldsymbol{X}^{l-1}+\boldsymbol{B}_{m}^{l}\right)
$$

in which $\boldsymbol{B}_{m}^{l}$ is the bias matrix. 


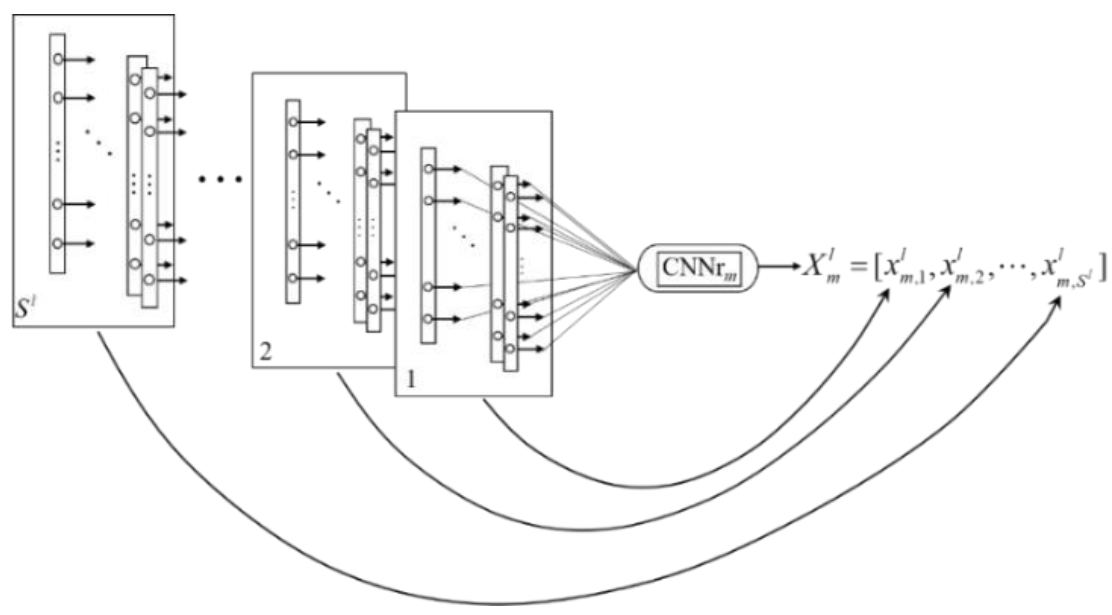

Fig. 1 Inputs and outputs of a virtual neuron

Since a kernel with a set of shared weights corresponds to an output of the virtual neuron, i.e., an output feature map, all $C^{l}$ kernels in the kernel bank generate $C^{l}$ outputs of the neuron, which are $C^{l}$ output feature maps on layer $l$. Fig. 2 shows the structure of the convolutional layer $l$, composed by virtual neurons $\mathrm{CNNr}_{1}, \ldots, \mathrm{CNNr}_{C^{l}}, \boldsymbol{X}^{l}=\left[\boldsymbol{X}_{1}^{l}, \boldsymbol{X}_{2}^{l}, \ldots, \boldsymbol{X}_{C^{\prime}}^{l}\right]$ is the output of the layer in question, then there is:

$$
\boldsymbol{X}^{l}=f\left(\boldsymbol{K}^{l} \otimes \boldsymbol{X}^{l-1}+\boldsymbol{B}^{l}\right)
$$

in which $\boldsymbol{B}^{l}$ is the bias matrix.

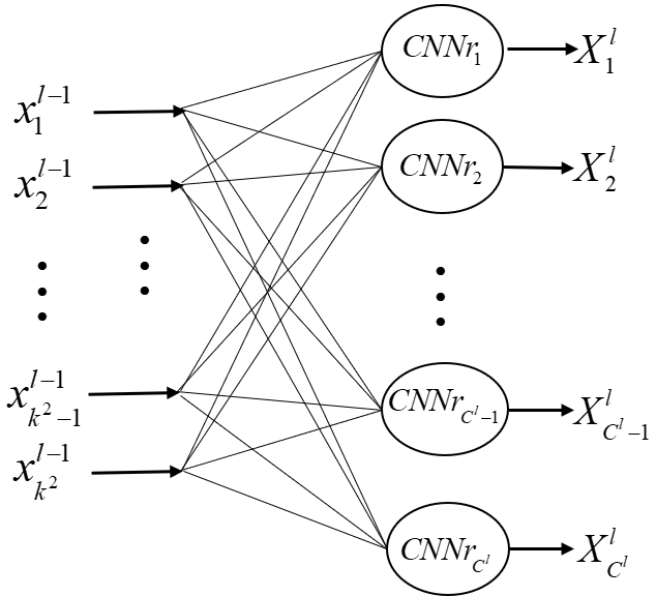

Fig. 2 The structure of a convolutional layer

Feature vectors extracted from the convolutional layers are fed to the fully-connected layers to accomplish discrimination. For the unity of forms, let $\boldsymbol{X}^{l-1}$ be the input to the fully-connected layer $l, K^{l}$ the coefficient matrix and $B^{l}$ the bias. The output $\boldsymbol{X}^{l}=\left[\boldsymbol{x}_{1}^{l}, \cdots, \boldsymbol{x}_{c^{l}}^{l}\right]$ of the layer can be expressed as follows:

$$
\boldsymbol{X}^{l}=f\left(\left(\boldsymbol{K}^{l}\right)^{\mathrm{T}} \boldsymbol{X}^{l-1}+\boldsymbol{B}^{l}\right)
$$

$\mathrm{T}$ means the operation of transposition. The dot product of the weight and input is written as $\boldsymbol{V}^{l}$ :

$$
\boldsymbol{V}^{l}=\left(\boldsymbol{K}^{l}\right)^{\mathrm{T}} \boldsymbol{X}^{l-1}
$$

Thus, formula (9) can be abbreviated as $\boldsymbol{X}^{l}=f\left(\boldsymbol{V}^{l}+\boldsymbol{B}^{l}\right)$. Therefore, in terms of the layer structure as shown in Fig. 2, the entire network can be described in a mode of full connection (Fig. 3), where $L r_{1}, \cdots, L r_{C^{t}}$ are neurons in the last fully-connected layer and $L$ is the number of total layers of the network. 


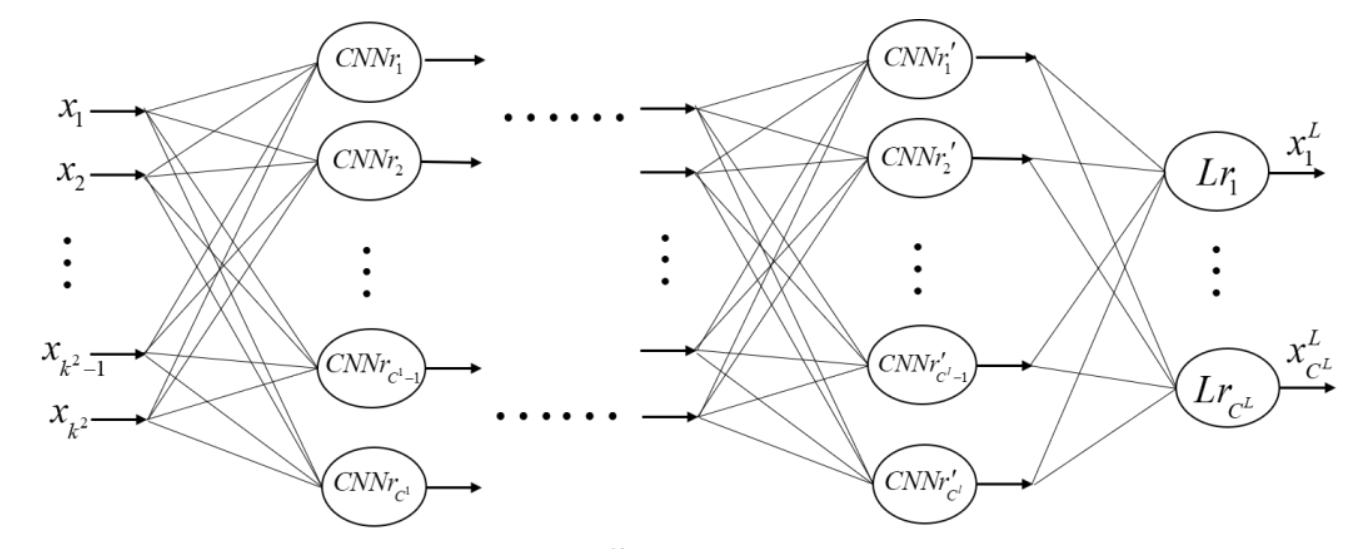

Input Layer

Intermediate Layer

Discriminant Layer

Fig. 3 The structure of the entire CNN

\section{Definitions of Sensitivity}

The output of a neural network depends on two key parameters: input and weight. Its output varies with perturbation to input or weight. Output variation of CNNs with the perturbation of parameters is a good indicator of fault tolerance, robustness or generalization of the network. This paper investigates CNNs' sensitivity to input perturbation by additive noises, which is useful for tackling image rotation, translation or scaling.

\subsection{Definitions for Convolutional Layers}

According to the output $\boldsymbol{X}_{m}^{l}$ of the neuron $\mathrm{CNNr}_{m}$ to the input $\boldsymbol{X}^{l-1}$ as given in formula (7), when the input is perturbed by $\Delta \boldsymbol{X}^{l-1}$, the output variation $\Delta \boldsymbol{X}_{m}^{l}$ is:

$$
\Delta \boldsymbol{X}_{m}^{l}=f\left(\boldsymbol{K}_{m}^{l} \otimes\left(\boldsymbol{X}^{l-1}+\Delta \boldsymbol{X}^{l-1}\right)+\boldsymbol{B}_{m}^{l}\right)-f\left(\boldsymbol{K}_{m}^{l} \otimes \boldsymbol{X}^{l-1}+\boldsymbol{B}_{m}^{l}\right)
$$

Instead of discussing the variation caused by a particular input perturbation, it is more meaningful to study the statistical variations of the output for assessing the network's performance. The mathematical expectation of the output variation of CNNs is considered in this paper. To avoid the offset of positives and negatives, we consider the squared variation.

Definition 1. (Sensitivity of a virtual neuron) For a given CNN with $L$ layers, $L_{c}$ convolutional layers and $L_{f}$ fully-connected layers, $L=L_{c}+L_{f}$. For a virtual neuron $\operatorname{CNNr}_{m}\left(m=1,2, \ldots, C^{l}\right)$ at convolutional layer $l\left(1 \leq l \leq L_{c}\right)$ with given input $\boldsymbol{X}^{l-1}$ and weight $\boldsymbol{K}_{m}^{l}$, its sensitivity is defined as the mathematical expectation of the squared output variation:

$$
\boldsymbol{S}_{m}^{l}=E\left[\left(f\left(\boldsymbol{K}_{m}^{l} \otimes\left(\boldsymbol{X}^{l-1}+\Delta \boldsymbol{X}^{l-1}\right)+\boldsymbol{B}_{m}^{l}\right)-f\left(\boldsymbol{K}_{m}^{l} \otimes \boldsymbol{X}^{l-1}+\boldsymbol{B}_{m}^{l}\right)\right)^{2}\right]
$$

$E(\cdot)$ is the mathematical expectation. Actually, $S_{m}^{l}$ is the sensitivity of the $m^{\text {th }}$ output feature map at layer $l$, which consists of sensitivities of all neurons on the map:

$$
\boldsymbol{S}_{m}^{l}=\left[\boldsymbol{s}_{m, 1}^{l}, \boldsymbol{s}_{m, 2}^{l}, \cdots, \boldsymbol{s}_{m, S^{l}}^{l}\right]
$$

Each component $s_{m, j}^{l}\left(j=1,2, \ldots, S^{l}\right)$ is:

$$
s_{m, j}^{l}=E\left\{\left[f\left(\sum_{i=1}^{C^{l-1}}\left(\hat{k}_{m, i}^{l}\right)^{T}\left(\hat{x}_{i, j}^{l-1}+\Delta \hat{x}_{i, j}^{l-1}\right)+\hat{b}_{m, j}^{l}\right)-f\left(\sum_{i=1}^{C^{l-1}}\left(\hat{k}_{m, i}^{l}\right)^{T} \hat{x}_{i, j}^{l-1}+\hat{b}_{m, j}^{l}\right)\right]^{2}\right\}
$$

where the hat notation in (14) means to stretch the item into a vector.

Definition 2. (Sensitivity of a convolutional layer) The sensitivity of a convolutional layer $l$ is defined as a vector containing all virtual neurons' sensitivity $\boldsymbol{S}_{m}^{l}$ due to the input perturbation $\Delta \boldsymbol{X}^{l-1}$ : 


$$
\boldsymbol{S}^{l}=\left[\boldsymbol{s}_{1}^{l}, \boldsymbol{s}_{2}^{l}, \cdots, \boldsymbol{s}_{C^{l}}^{l}\right]
$$

Simply, the sensitivity of a convolution layer can be expressed as:

$$
\boldsymbol{S}^{l}=E\left[\left(f\left(\boldsymbol{K}^{l} \otimes\left(\boldsymbol{X}^{l-1}+\Delta \boldsymbol{X}^{l-1}\right)+\boldsymbol{B}^{l}\right)-f\left(\boldsymbol{K}^{l} \otimes \boldsymbol{X}^{l-1}+\boldsymbol{B}^{l}\right)\right)^{2}\right]
$$

\subsection{Definitions for Fully-connected Layers}

The feature map of the last convolutional layer is flattened as vectors as the input of the fullyconnected layers. By fully-connection to the neurons in the hidden layers, the output is propagated forward and the error is back-propagated in the fully-connected layers with a similar structure to a MLP. Therefore, we define the sensitivity of a fully-connected layer in the same way as an MLP. Supposing that there are $C^{l}$ neurons in a fully-connected layer $l\left(L_{C}+1 \leq l \leq L\right), X^{l-1}$ is the input of the $m^{\text {th }}$ neuron $L r_{m}, \Delta \boldsymbol{X}^{l-1}$ is the input perturbation, and $\boldsymbol{K}_{m}^{l}$ is the weight, then the output of the $m^{\text {th }}$ neuron is:

$$
x_{m}^{l}=f\left(\left(\boldsymbol{K}_{m}^{l}\right)^{\mathrm{T}} \boldsymbol{X}^{l-1}+b_{m}^{l}\right)
$$

After perturbation, the output variation of the neuron is:

$$
\Delta x_{m}^{l}=f\left(\left(\boldsymbol{K}_{m}^{l}\right)^{\mathrm{T}}\left(\boldsymbol{X}^{l-1}+\Delta \boldsymbol{X}^{l-1}\right)+b_{m}^{l}\right)-f\left(\left(\boldsymbol{K}_{m}^{l}\right)^{\mathrm{T}} \boldsymbol{X}^{l-1}+b_{m}^{l}\right)
$$

$b_{m}^{l}$ is a bias. The output of the layer is denoted as $\boldsymbol{X}^{l}$ which comprises all the output neurons $\boldsymbol{X}^{l}=\left[x_{1}^{l}, x_{2}^{l}, \cdots, x_{c^{l}}^{l}\right]$. Mathematically, $\boldsymbol{X}^{l}$ can be written as:

$$
\boldsymbol{X}^{l}=f\left(\left(\boldsymbol{K}^{l}\right)^{\mathrm{T}} \boldsymbol{X}^{l-1}+\boldsymbol{B}^{l}\right)
$$

Where $\boldsymbol{K}^{l}=\left[K_{1}^{l}, K_{2}^{l}, \cdots, K_{C^{l}}^{l}\right]$ is the weight matrix in the layer. Due to the perturbation $\Delta \boldsymbol{X}^{l-1}$, the output variation $\Delta \boldsymbol{X}^{l}$ is:

$$
\Delta \boldsymbol{X}^{l}=f\left(\left(\boldsymbol{K}^{l}\right)^{\mathrm{T}}\left(\boldsymbol{X}^{l-1}+\Delta \boldsymbol{X}^{l-1}\right)+\boldsymbol{B}^{l}\right)-f\left(\left(\boldsymbol{K}^{l}\right)^{\mathrm{T}} \boldsymbol{X}^{l-1}+\boldsymbol{B}^{l}\right)
$$

In the same way as convolutional layers, here we also use the mathematical expectation to define the sensitivity of fully-connected layers.

Definition 3. (Sensitivity of a neuron) For a neuron $L r_{m}\left(m=1,2, \ldots, C^{l}\right)$ in a fully-connected layer $l\left(L_{c}+1 \leq l \leq L\right)$ with given input $\boldsymbol{X}^{l-1}$ and weight $\boldsymbol{K}_{m}^{l}$, its sensitivity is defined as the mathematical expectation of the squared output variation:

$$
\boldsymbol{S}_{m}^{l}=E\left[\left(f\left(\left(\boldsymbol{K}_{m}^{l}\right)^{\mathrm{T}}\left(\boldsymbol{X}^{l-1}+\Delta \boldsymbol{X}^{l-1}\right)+b_{m}^{l}\right)-f\left(\left(\boldsymbol{K}_{m}^{l}\right)^{\mathrm{T}} \boldsymbol{X}^{l-1}+b_{m}^{l}\right)\right)^{2}\right]
$$

Definition 4. (Sensitivity of a fully-connected layer) The sensitivity of a layer $l$ is defined as a vector containing all neurons' sensitivity due to input perturbation $\Delta \boldsymbol{X}^{l-1}$ :

$$
S^{l}=\left[s_{1}^{l}, s_{2}^{l}, \cdots, s_{C^{l}}^{l}\right]
$$

The sensitivity of layer $l$ can be denoted as:

$$
\boldsymbol{S}^{l}=E\left[\left(f\left(\left(\boldsymbol{K}^{l}\right)^{\mathrm{T}}\left(\boldsymbol{X}^{l-1}+\Delta \boldsymbol{X}^{l-1}\right)+\boldsymbol{B}^{l}\right)-f\left(\left(\boldsymbol{K}^{l}\right)^{\mathrm{T}} \boldsymbol{X}^{l-1}+\boldsymbol{B}^{l}\right)\right)^{2}\right]
$$

For a given $\mathrm{CNN} S^{\mathrm{CNN}}$ with input $\boldsymbol{X}^{\mathbf{0}}$ and weight $\boldsymbol{K}^{\mathbf{0}}$, its sensitivity to input perturbation is a vector that consists of each neuron's sensitivity $S_{1}^{L}, \cdots, S_{C^{L}}^{L}$ in the final fully-connected layer $L$, $S^{\mathrm{CNN}}=\left[S_{1}^{L}, \cdots, S_{C^{L}}^{L}\right]$. Then, the sensitivity of the whole network $S^{\mathrm{CNN}}$ is:

$$
\boldsymbol{S}^{\mathrm{CNN}}=E\left[\left(f\left(\left(\boldsymbol{K}^{L}\right)^{\mathrm{T}}\left(\boldsymbol{X}^{L-1}+\Delta \boldsymbol{X}^{L-1}\right)+\boldsymbol{B}^{L}\right)-f\left(\left(\boldsymbol{K}^{L}\right)^{\mathrm{T}} \boldsymbol{X}^{L-1}+\boldsymbol{B}^{L}\right)\right)^{2}\right]
$$

\section{Computation of a CNN's Sensitivity}

Based on the characteristic of forward propagation, the sensitivity of a CNN can be computed layer by layer. Theoretically, the mathematical expectation of sensitivity can be computed by an integration. Taking an individual neuron $\mathrm{CNNr}_{m}$ in the convolutional layer for example, its sensitivity can be found by an integration on the space of a local receptive field:

$$
S_{m}^{l}=\int \cdots \int\left(\Delta X_{m}^{l}\right)^{2} d \Delta x_{1}^{l-1} d \Delta x_{2}^{l-1} \cdots d \Delta x_{k^{2}}^{l-1}
$$

This is a high-dimensional integration, which is hard to obtain the accurate result in practical computation. Similarly, the sensitivity of the first fully-connected layer can be computed by an 
integration in its input space, which is formed by flattening feature maps on the last convolution layer. A higher dimensional integration is required:

$$
S_{m}^{L_{c}+1}=\int \cdots \int\left(\Delta X_{m}^{L_{c}+1}\right)^{2} d \Delta x_{1}^{L_{c}} d \Delta x_{2}^{L_{c}} \cdots d \Delta x_{C^{L_{c}} \times H^{L_{c}} \times W^{L_{c}}}^{L_{c}}
$$

To avoid the above complicated high-dimensional integration, we reconsider the sensitivity problem from another angle. Essentially, the operation of convolution on the convolutional layers can be seen as a weighted sum of convolution kernel coefficients and elements in the local receptive field of inputs, while the inner product on the fully-connected layer is also the weighted sum between the input vector and the connected weights. According to the Central Limit Theorem, the sum of independent and identically distributed random variables obeys the normal distribution. The results after convolution or inner product can be approximated by a normal distribution on the assumption that the input components are independent from each other at each layer. Taking the results after convolution or inner product as a whole, it obeys the normal distribution approximately. The parameters for the normal distribution can be determined according to the distribution of the initial input perturbation. Thus the mathematical expectation of sensitivity can be found by the integration in numerical. The detailed procedure for approximating sensitivity on convolutional and fully- connected layers is given below.

(1) The Convolutional Layer

For a given CNN, let $\boldsymbol{X}^{0}$ be the input, $\Delta \boldsymbol{X}^{0}$ input perturbation with known distribution, and $\boldsymbol{K}^{1}$ the kernel weight at the first convolution layer, then the convolved input perturbation can be denoted as $\boldsymbol{V}^{1}$ :

$$
\boldsymbol{V}^{1}=\boldsymbol{K}^{1} \otimes \Delta \boldsymbol{X}^{0}
$$

With the assumption as mentioned above, $\boldsymbol{V}^{1}$ obeys the normal distribution $\boldsymbol{V}^{1} \sim N\left(\mu_{1}, \sigma_{1}^{2}\right)$, among which $\mu_{1}$ is the mathematical expectation of $\boldsymbol{V}^{1}$ and $\sigma_{1}^{2}$ is the variance. Based on the properties of the normal distribution, $\mu_{1}$ and $\sigma_{1}^{2}$ can be determined by the distribution of $\Delta \boldsymbol{X}^{0}$ :

$$
\begin{gathered}
\mu_{1}=E\left(\boldsymbol{V}^{1}\right)=\boldsymbol{K}^{1} \otimes E\left(\Delta \boldsymbol{X}^{0}\right) \\
\sigma_{1}^{2}=D\left(\boldsymbol{V}^{1}\right)=\left(\boldsymbol{K}^{1}\right)^{2} \otimes D\left(\Delta \boldsymbol{X}^{0}\right)
\end{gathered}
$$

$E($.$) is the mathematical expectation, D\left(\right.$.) the variance, and $\left(\boldsymbol{K}^{1}\right)^{2}$ the squared weight coefficients. Then the sensitivity of the first convolution layer can be found by integrating $\boldsymbol{V}^{1}$ :

$$
\boldsymbol{S}^{1}=\int\left[\left(f\left(\boldsymbol{K}^{1} \otimes \boldsymbol{X}^{0}+\boldsymbol{V}^{1}+\boldsymbol{B}^{1}\right)-f\left(\boldsymbol{K}^{1} \otimes \boldsymbol{X}^{0}+\boldsymbol{B}^{1}\right)\right)^{2}\right] \varphi\left(\boldsymbol{V}^{1}\right) d \boldsymbol{V}^{1}
$$

$\varphi\left(\boldsymbol{V}^{1}\right)$ is the density function of $\boldsymbol{V}^{1}$, with the function expression:

$$
\varphi(x)=\frac{1}{\sqrt{2 \pi} \sigma} \exp \left(-\frac{(x-\mu)^{2}}{2 \sigma^{2}}\right)
$$

among which $\mu$ and $\sigma^{2}$ are parameters, $\mu=\mu_{1}, \sigma^{2}=\sigma_{1}^{2}$ in $\varphi\left(V^{1}\right)$. Thus, the sensitivity of the first convolutional layer which is directly connected to the input layer can be found by a simple onedimensional integration, instead of a complex multi-integration. Sensitivity $S^{1}$ is the output variation $\Delta \boldsymbol{X}^{1}$ of the current layer, which corresponds to the input perturbation of the next layer. However, unlike the first layer, the distribution of input perturbation $\Delta \boldsymbol{X}^{l-1}$ for the second convolutional layer is unknown, so it is difficult to compute its sensitivity in the same way as for the first layer.

From the second convolutional layer on, the perturbed input $\left(\boldsymbol{X}^{l-1}+\Delta \boldsymbol{X}^{l-1}\right)$ is considered as a whole random variable, denoted as $\tilde{\boldsymbol{Y}}^{l-1}$. With the assumption that the components are independent and identically distributed, the convolved input $\boldsymbol{V}^{l}$ approximately obeys the normal distribution:

$$
\boldsymbol{V}^{l}=\boldsymbol{K}^{l} \otimes \tilde{\boldsymbol{Y}}^{l-1} \sim N\left(\mu_{l}, \sigma_{l}^{2}\right)
$$


$\mu_{l}$ is the mathematical expectation of $V^{l}$ and $\sigma_{l}^{2}$ is its variance. According to the properties of the normal distribution, the following holds:

$$
\begin{aligned}
\mu_{l} & =E\left(\boldsymbol{V}^{l}\right)=\boldsymbol{K}^{l} \otimes E \tilde{\boldsymbol{Y}}^{l-1} \\
\sigma_{l}^{2} & =D\left(\boldsymbol{V}^{l}\right)=\left(\boldsymbol{K}^{l}\right)^{2} \otimes D \tilde{\boldsymbol{Y}}^{l-1}
\end{aligned}
$$

$\left(\boldsymbol{K}^{l}\right)^{2}$ means squared weight coefficients. Therefore, as long as $E \tilde{\boldsymbol{Y}}^{l-1}$ and $D \tilde{\boldsymbol{Y}}^{l-1}$ are determined, $\varphi\left(\boldsymbol{V}^{l}\right)$ is decided, by which the sensitivity of the layer can be computed by the following integration:

$$
\boldsymbol{S}^{l}=\int\left[\left(f\left(\boldsymbol{V}^{l}+\boldsymbol{B}^{l}\right)-f\left(\boldsymbol{K}^{l} \otimes \boldsymbol{X}^{l-1}+\boldsymbol{B}^{l}\right)\right)^{2}\right] \varphi\left(\boldsymbol{V}^{l}\right) d \boldsymbol{V}^{l}
$$

In this way, the problem of finding the sensitivity of layer $l$ is transformed into finding $E \tilde{\mathbf{Y}}^{l-1}$ and $D \tilde{\boldsymbol{Y}}^{l-1}$. For forward propagations, $\tilde{\boldsymbol{Y}}^{l-1}$ is just the perturbed output of layer $l-1$, which can be obtained after convolution and transfer functions:

$$
\tilde{\boldsymbol{Y}}^{l-1}=f\left(\boldsymbol{K}^{l-1} \otimes\left(\boldsymbol{X}^{l-2}+\Delta \boldsymbol{X}^{l-2}\right)+\boldsymbol{B}^{l-1}\right)
$$

$\left(\boldsymbol{X}^{l-2}+\Delta \boldsymbol{X}^{l-2}\right)$ is the input of layer $l-1$, i.e., the perturbed output of layer $l-2$, written as $\tilde{\boldsymbol{Y}}^{l-2}$. The convolved result of $\tilde{\boldsymbol{Y}}^{l-2}$ (denoted as $\boldsymbol{V}^{l-1}$ ) is:

$$
\boldsymbol{V}^{l-1}=\boldsymbol{K}^{l-1} \otimes \tilde{\boldsymbol{Y}}^{l-2}
$$

Then, Formula (36) can be rewritten as:

$$
\tilde{\boldsymbol{Y}}^{l-1}=f\left(\boldsymbol{V}^{l-1}+\boldsymbol{B}^{l-1}\right)
$$

Thus, $E \tilde{\boldsymbol{Y}}^{l-1}$ and $D \tilde{\boldsymbol{Y}}^{l-1}$ can be found by integrating $\boldsymbol{V}^{l-1}$ :

$$
\begin{gathered}
E \tilde{\boldsymbol{Y}}^{l-1}=\int f\left(\boldsymbol{V}^{l-1}+\boldsymbol{B}^{l-1}\right) \varphi\left(\boldsymbol{V}^{l-1}\right) d\left(\boldsymbol{V}^{l-1}\right) \\
D \tilde{\boldsymbol{Y}}^{l-1}=\int\left[f\left(\boldsymbol{V}^{l-1}+\boldsymbol{B}^{l-1}\right)\right]^{2} \varphi\left(\boldsymbol{V}^{l-1}\right) d\left(\boldsymbol{V}^{l-1}\right)-\left[\int f\left(\boldsymbol{V}^{l-1}+\boldsymbol{B}^{l-1}\right) \varphi\left(\boldsymbol{V}^{l-1}\right) d\left(\boldsymbol{V}^{l-1}\right)\right]^{2}
\end{gathered}
$$

To find $E \tilde{\boldsymbol{Y}}^{l-1}$ and $D \tilde{\boldsymbol{Y}}^{l-1}$ is then transformed into determining the density function $\varphi\left(\boldsymbol{V}^{l-1}\right)$ of layer $l-1$, or determining $E\left(V^{l-1}\right)$ and $D\left(V^{l-1}\right)$. By (37), we have:

$$
\begin{gathered}
E\left(\boldsymbol{V}^{l-1}\right)=\boldsymbol{K}^{l-1} \otimes E \tilde{\boldsymbol{Y}}^{l-2} \\
D\left(\boldsymbol{V}^{l-1}\right)=\left(\boldsymbol{K}^{l-1}\right)^{2} \otimes D \tilde{\boldsymbol{Y}}^{l-2}
\end{gathered}
$$

In order to get the values for $E\left(\boldsymbol{V}^{l-1}\right)$ and $D\left(\boldsymbol{V}^{l-1}\right)$, we need to know the values of $E \tilde{\boldsymbol{Y}}^{l-2}$ and $D \tilde{\boldsymbol{Y}}^{l-2}$.

To compute the sensitivity of layer $l$, we need to know the density function $\varphi\left(V^{l}\right)$ first. Parameters $E \boldsymbol{V}^{l}$ and $D \boldsymbol{V}^{l}$ in $\varphi\left(\boldsymbol{V}^{l}\right)$ can be found by the mathematical expectation $E \tilde{\boldsymbol{Y}}^{\tilde{u}^{-1}}$ and variance $D \tilde{\boldsymbol{Y}}^{l-1}$ of the perturbed output $\tilde{\boldsymbol{Y}}^{l-1}$ of the former layer (l-1). To obtain $E \tilde{\boldsymbol{Y}}^{l-1}$ and $D \tilde{\boldsymbol{Y}}^{l-1}$, the density function of the former layer $\varphi\left(\boldsymbol{V}^{l-1}\right)$ is needed for the integration, which is determined by parameters $E \boldsymbol{V}^{l-1}$ and $D \boldsymbol{V}^{l-1}$. Similarly, $E \boldsymbol{V}^{l-1}$ and $D \boldsymbol{V}^{l-1}$ can be found through $E \tilde{\boldsymbol{Y}}^{l-2}$ and $D \tilde{\boldsymbol{Y}}^{l-2}$, the expectation and variance of the perturbed output of layer $l-2$. Thus it propagates back layer by layer until $E \tilde{\boldsymbol{Y}}^{1}$ and $D \tilde{\boldsymbol{Y}}^{1}$ of the first convolution layer. Taking $E \tilde{\boldsymbol{Y}}^{1}$ and $D \tilde{\boldsymbol{Y}}^{1}$ as initial values, the density function $\varphi\left(\boldsymbol{V}^{l}\right)$ of the convolved input of each layer can be determined, and the sensitivity of each layer $S^{l}$ is then obtained by (35). $E \tilde{\boldsymbol{Y}}^{l}$ and $D \tilde{\boldsymbol{Y}}^{l}$ can be found iteratively:

$$
\begin{gathered}
E \tilde{\boldsymbol{Y}}^{l}=\int f\left(\boldsymbol{V}^{l}+\boldsymbol{B}^{l}\right) \varphi\left(\boldsymbol{V}^{l}\right) d\left(\boldsymbol{V}^{l}\right) \\
D \tilde{\boldsymbol{Y}}^{l}=\int\left[f\left(\boldsymbol{V}^{l}+\boldsymbol{B}^{l}\right)\right]^{2} \varphi\left(\boldsymbol{V}^{l}\right) d\left(\boldsymbol{V}^{l}\right)-\left[\int f\left(\boldsymbol{V}^{l}+\boldsymbol{B}^{l}\right) \varphi\left(\boldsymbol{V}^{l}\right) d\left(\boldsymbol{V}^{l}\right)\right]^{2}
\end{gathered}
$$

Based on the given distribution of the input perturbation $\Delta \boldsymbol{X}^{0}$, the distribution of $\boldsymbol{V}^{1}$ can be determined by $\boldsymbol{V}^{1} \sim N\left(\mu_{1}, \sigma_{1}^{2}\right), \quad \mu_{1}=\boldsymbol{K}^{1} \otimes E\left(\Delta \boldsymbol{X}^{0}\right) \quad$ and $\sigma_{1}^{2}=\left(\boldsymbol{K}^{1}\right)^{2} \otimes D\left(\Delta \boldsymbol{X}^{0}\right)$. So the initial values $E \tilde{\boldsymbol{Y}}^{1}$ and $D \tilde{\boldsymbol{Y}}^{1}$ for the iterative process are:

$$
\begin{aligned}
E \tilde{\boldsymbol{Y}}^{1} & =E\left[f\left(\boldsymbol{K}^{1} \otimes\left(\boldsymbol{X}^{0}+\Delta \boldsymbol{X}^{0}\right)\right)\right] \\
& =\int f\left(\boldsymbol{K}^{1} \otimes \boldsymbol{X}^{0}+\boldsymbol{V}^{1}+\boldsymbol{B}^{1}\right) \varphi\left(\boldsymbol{V}^{1}\right) d \boldsymbol{V}^{1} \\
D \tilde{\boldsymbol{Y}}^{1} & =E\left(\tilde{\boldsymbol{Y}}^{1}\right)^{2}-\left(E \tilde{\boldsymbol{Y}}^{1}\right)^{2}
\end{aligned}
$$




$$
=\int\left[f\left(\boldsymbol{K}^{1} \otimes \boldsymbol{X}^{0}+\boldsymbol{V}^{1}+\boldsymbol{B}^{1}\right)\right]^{2} \varphi\left(\boldsymbol{V}^{1}\right) d \boldsymbol{V}^{1}-\left[\int f\left(\boldsymbol{K}^{1} \otimes \boldsymbol{X}^{0}+\boldsymbol{V}^{1}+\boldsymbol{B}^{1}\right) \varphi\left(\boldsymbol{V}^{1}\right) d \boldsymbol{V}^{1}\right]^{2}
$$

By iterating on the initial values, the density function and sensitivity of each layer can be determined layer by layer until the last convolutional layer.

(2) The Fully-connected Layer

The feature maps at the convolutional layer are flattened as input vectors of the whole fullyconnected layers, and the output is obtained through the transfer function after the inner product with corresponding weights. According to the previous approximation, the inner product of weights and input vectors also obeys the normal distribution. To unify the symbols, $\tilde{\boldsymbol{Y}}^{l-1}$ is also used to represent the perturbed output $\left(\boldsymbol{X}^{l-1}+\Delta \boldsymbol{X}^{l-1}\right)$ at layer $l-1\left(L_{C}+1 \leq l \leq L\right)$, i.e., the perturbed input at layer $l$, and $\boldsymbol{V}^{l}$ is the inner product of the input and weights, $\boldsymbol{V}^{l}=\left(\boldsymbol{K}^{l}\right)^{\mathrm{T}} \tilde{\boldsymbol{Y}}^{l-1}$. Assuming that the components of $\tilde{\boldsymbol{Y}}^{l-1}$ are independent of each other, $\boldsymbol{V}^{l}$ meets:

$$
\boldsymbol{V}^{l} \sim N\left(\left(\boldsymbol{K}^{l}\right)^{\mathrm{T}} E \tilde{\boldsymbol{Y}}^{l-1}, \boldsymbol{K}^{l} D \tilde{\boldsymbol{Y}}^{l-1}\left(\boldsymbol{K}^{l}\right)^{\mathrm{T}}\right)
$$

Taking the same iterative procedure as for convolutional layers, the sensitivity of the fullyconnected layers can be computed on the same assumption of the approximate normal distribution. The initial iterative value is the perturbed output $\tilde{\boldsymbol{Y}}^{L_{C}+1}$ of the first fully-connected layer (layer $L_{C}$ +1 ), which is connected to the last convolutional layer (layer $\left.L_{C}\right) . E\left(\tilde{\boldsymbol{Y}}^{L_{c}+1}\right)$ and $D\left(\tilde{\boldsymbol{Y}}^{L_{c}+1}\right)$ can be found by the distribution of the inner product $\boldsymbol{V}^{L_{c}+1}$ of the weight $\boldsymbol{K}^{L_{c}+1}$ with the perturbed input $\tilde{\boldsymbol{Y}}^{L_{C}}$ of the layer:

$$
\begin{aligned}
E\left(\tilde{\boldsymbol{Y}}^{L_{C}+1}\right)= & E\left[f\left(\left(\boldsymbol{K}^{L_{C}+1}\right)^{\mathrm{T}} \tilde{\boldsymbol{Y}}^{L_{C}}+\boldsymbol{B}^{L_{C}+1}\right)\right] \\
& =\int f\left(\boldsymbol{V}^{L_{C}+1}+\boldsymbol{B}^{L_{C}+1}\right) \varphi\left(\boldsymbol{V}^{L_{C}+1}\right) d \boldsymbol{V}^{L_{C}+1} \\
D\left(\tilde{\boldsymbol{Y}}^{L_{C}+1}\right)= & E\left(\tilde{\boldsymbol{Y}}^{L_{C}+1}\right)^{2}-\left(E \tilde{\boldsymbol{Y}}^{L_{C}+1}\right)^{2} \\
& =\int\left[f\left(\boldsymbol{V}^{L_{C}+1}+\boldsymbol{B}^{L_{C}+1}\right)\right]^{2} \varphi\left(\boldsymbol{V}^{L_{C}+1}\right) d \boldsymbol{V}^{L_{C}+1}-\left(E \tilde{\boldsymbol{Y}}^{L_{C}+1}\right)^{2}
\end{aligned}
$$

among which $\varphi\left(\boldsymbol{V}^{L_{c}+1} ; E\left(\boldsymbol{V}^{L_{c}+1}\right), D\left(\boldsymbol{V}^{L_{c}+1}\right)\right)$ is the probability density function. Both parameters $E\left(\boldsymbol{V}^{L_{c}+1}\right)$ and $D\left(\boldsymbol{V}^{L_{c}+1}\right)$ can be found by $\tilde{\boldsymbol{Y}}^{L_{c}}$ :

$$
\begin{gathered}
E\left(\boldsymbol{V}^{L_{C}+1}\right)=\left(\boldsymbol{K}^{L_{C}+1}\right)^{\mathrm{T}} E \tilde{\boldsymbol{Y}}^{L_{C}} \\
D\left(\boldsymbol{V}^{L_{C}+1}\right)=\boldsymbol{K}^{L_{C}+1} D \tilde{\boldsymbol{Y}}^{L_{C}}\left(\boldsymbol{K}^{L_{C}+1}\right)^{\mathrm{T}}
\end{gathered}
$$

While $E \tilde{\boldsymbol{Y}}^{L_{C}}$ and $D \tilde{\boldsymbol{Y}}^{L_{C}}$ are computed layer by layer for all the convolutional layers. Then, according to (48) and (49), $E \tilde{\boldsymbol{Y}}^{L_{c}+1}$ and $D \tilde{\boldsymbol{Y}}^{L_{c}+1}$ are obtained, and as the initial values to find $E \boldsymbol{V}^{l}$ and $D \boldsymbol{V}^{l}$ at each fully-connected layer by the iteration:

$$
\begin{aligned}
E\left(\boldsymbol{V}^{l}\right) & =\left(\boldsymbol{K}^{l}\right)^{\mathrm{T}} E \tilde{\boldsymbol{Y}}^{l-1} \\
D\left(\boldsymbol{V}^{l}\right) & =\boldsymbol{K}^{l} D \tilde{\boldsymbol{Y}}^{l-1}\left(\boldsymbol{K}^{l}\right)^{\mathrm{T}}
\end{aligned}
$$

Therefore, the density function $\varphi\left(\boldsymbol{V}^{l} ; E \boldsymbol{V}^{l}, D \boldsymbol{V}^{l}\right)$ can be determined, and the sensitivity of each layer $S^{l}$ is:

$$
\boldsymbol{S}^{l}=\int\left[\left(f\left(\boldsymbol{V}^{l}+\boldsymbol{B}^{l}\right)-f\left(\left(\boldsymbol{K}^{l}\right)^{\mathrm{T}} \boldsymbol{X}^{l-1}+\boldsymbol{B}^{l}\right)\right]^{2} \varphi\left(\boldsymbol{V}^{l}\right) d \boldsymbol{V}^{l}\right.
$$

Furthermore, $E \tilde{\boldsymbol{Y}}^{l}$ and $D \tilde{\boldsymbol{Y}}^{l}$ can be found by integrals:

$$
\begin{gathered}
E \tilde{\boldsymbol{Y}}^{l}=\int f\left(\boldsymbol{V}^{l}+\boldsymbol{B}^{l}\right) \varphi\left(\boldsymbol{V}^{l}\right) d\left(\boldsymbol{V}^{l}\right) \\
D \tilde{\boldsymbol{Y}}^{l}=\int\left[f\left(\boldsymbol{V}^{l}+\boldsymbol{B}^{l}\right)\right]^{2} \varphi\left(\boldsymbol{V}^{l}\right) d\left(\boldsymbol{V}^{l}\right)-\left[\int f\left(\boldsymbol{V}^{l}+\boldsymbol{B}^{l}\right) \varphi\left(\boldsymbol{V}^{l}\right) d\left(\boldsymbol{V}^{l}\right)\right]^{2}
\end{gathered}
$$

Then the density function $\varphi\left(\boldsymbol{V}^{l+1} ; E \boldsymbol{V}^{l+1}, D \boldsymbol{V}^{l+1}\right)$ of the perturbed input $\boldsymbol{V}^{l+1}$ and the sensitivity of the next layer can be determined by (52)-(54), until the sensitivity of the last layer (layer $L$ ) $S^{L}$, which is also defined as the sensitivity of the whole $\mathrm{CNN}, S^{\mathrm{CNN}}=S^{L}$. In order to provide a clear picture in determining the sensitivity of the entire $\mathrm{CNN}$, a complete calculating algorithm is presented as follows: 


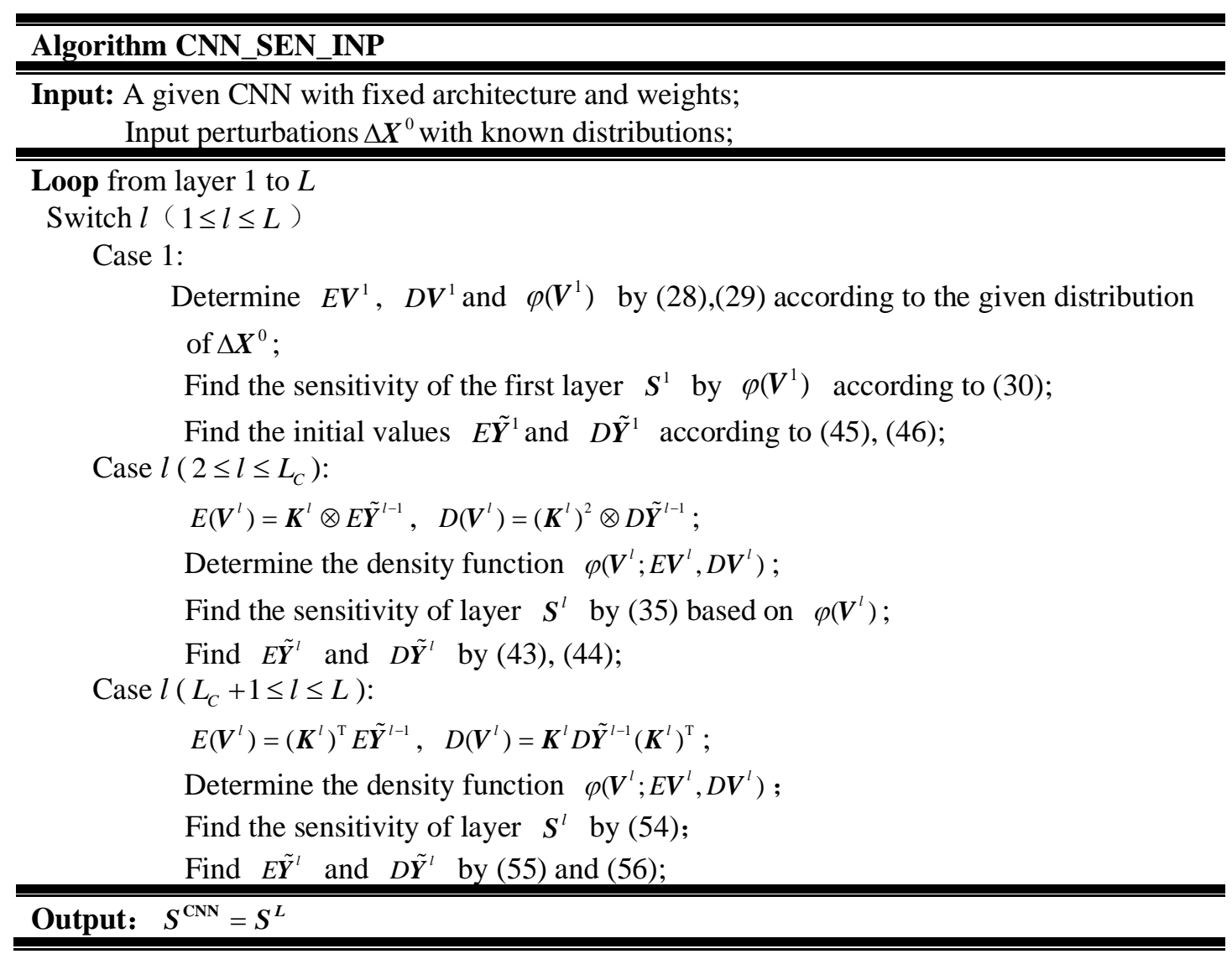

\section{Experimental verifications}

\subsection{Experiments}

In this section, we report a series of experiments conducted to verify the reasonability of the definitions and feasibility of the approximating algorithm proposed. By considering a typical and simple CNN, we compute its sensitivity to additive noises. The network is set as $\mathrm{C} 1(6,5,5)-\mathrm{P} 1(2,2)-$ C2(12,5,5)- $\mathrm{P} 2(2,2)-\mathrm{F}(192)-\mathrm{O}(10)$ denoted as $\mathrm{CNN}-1$, in which $\mathrm{C}$ means a convolution layer, $\mathrm{P}$ a pooling layer, $\mathrm{F}$ a fully-connected layer, and $\mathrm{O}$ an output layer. The number inside a pair of brackets indicates the number of parameters used for that given type of layer. For example, $\mathrm{C} 1(6,5,5)$ means 6 kernels with a size of $5 \times 5$ on the convolutional layer $\mathrm{C} 1$, while $\mathrm{F}(192)$ means that there are 192 neurons on the fully-connected layer F. The parameters in the model are obtained by pre-training on randomly generated initial values for 5 times. Sigmoid is set as the transfer function. To ensure the reproducibility, the publicly available dataset MNIST is used with a training accuracy of $95.32 \%$. The dataset contains 70,000 gray-scale images of handwritten numbers with a size of $28 \times 28$, 60,000 training samples and 10,000 test samples, among which the training samples can be further divided into 55,000 training samples and 5,000 verification samples.

To demonstrate the validity of the proposed algorithm, we compare the simulation results with the theoretically computed sensitivity. On the given $\mathrm{CNN}$, the input perturbation can cause variations of the corresponding output. Simulation sensitivity is defined as the average variation before and after perturbation, which is denoted as SS. On the other hand, the theoretical sensitivity is computed by the algorithm CNN_SEN_INP, denoted as TS. In the following experiments, SS is obtained by averaging the output variations of 1000 randomly selected test samples. TS is calculated under the same distribution of perturbations. The proximity between $T S$ and $S S$ can be used to show the estimation accuracy of the algorithm. 
As mentioned above that one virtual neuron corresponds to one feature map, the output of a virtual neuron is all the values on the output feature map generated by a convolution kernel sliding over the input feature map. The difference between $T S$ and $S S$ for a virtual neuron is measured by the distance between the feature map matrices of the two types of sensitivity. The Chi-square distance is used in this paper to measure the proximity between $T S$ and $S S$. It is defined as:

$$
\chi^{2}(T S, S S)=\frac{1}{2} \sum \frac{\left(T S_{i}-S S_{i}\right)^{2}}{T S_{i}+S S_{i}}
$$

where $T S$ and $S S$ denote the theoretical sensitivity and simulation sensitivity respectively. The closer the Chi-square distance is to zero, the more precise is the theoretical sensitivity.

Because intensive noises can affect the output of the network in a moderate or dramatic manner, this paper attempts to verify the feasibility of the sensitivity algorithm to noises with various distributions and at various intensity levels. Four distributions of noises: Gaussian, Uniform, Salt \& Pepper, and Rayleigh are considered in the simulating experiments to demonstrate the effectiveness of the sensitivity algorithm for different forms of additive noises. In addition, various parameter values for the distributions are set to simulate different levels of noise perturbations for different distributions of noises.

Table 1 lists the probability density functions, parameters, the mathematical expectations $E X$ and variances $D X$ of four types of noises. Taking the most commonly used Gaussian distribution as an example, parameter $a$ varies from 0 to 0.5 with a step of 0.1 and $b$ is set to $0.02,0.08,0.1,0.3,0.5$, 0.8 correspondingly. According to different parameter values of $a$ and $b, E X$ and $D X$ of the distribution are determined and listed in the last column of the table, which are initial values in the iterative algorithm of finding the sensitivity.

Table 1 Probability Density Functions, parameters, expectations and variances of the noise obeying Gaussian, uniform, salt \& pepper, and Rayleigh distributions

\begin{tabular}{|c|c|c|c|c|}
\hline Distribution & \multicolumn{2}{|c|}{ (Probability Density Function) } & $(a, b)$ & $E X, D X$ \\
\hline Gaussian & $p(x)=\frac{1}{\sqrt{2 \pi} b} e^{-(x-a)^{2} /}$ & & $\begin{array}{l}(0,0.02) \\
(0.1,0.08) \\
(0.2,0.1) \\
(0.3,0.3) \\
(0.4,0.5) \\
(0.5,0.8) \\
\end{array}$ & $\begin{aligned} E X & =a \\
D X & =b^{2}\end{aligned}$ \\
\hline Uniform & $p(x)=\left\{\begin{array}{c}\frac{1}{b-a} \\
0\end{array}\right.$ & & $\begin{array}{c}(0,1) \\
(0,0.5) \\
(0.2,0.8) \\
(0.3,0.7) \\
(0.5,1) \\
\end{array}$ & $\begin{array}{c}E X=\frac{a+b}{2} \\
D X=\frac{(b-a)^{2}}{12}\end{array}$ \\
\hline Salt \& Pepper & $p(x)=\left\{\begin{array}{cc}p_{a} & z=a \\
p_{b} & z=b \\
0 & \text { others }\end{array}\right.$ & & $\begin{array}{c}(0.05,0.05) \\
(0.1,0.1) \\
(0.2,0.2) \\
(0.4,0.4) \\
(0.5,0.5) \\
\end{array}$ & $\begin{array}{c}E X=a p_{a}+b p_{b} \\
D X=(a-m)^{2} p_{a}+(b-m)^{2} p_{b}\end{array}$ \\
\hline Rayleigh & $p(x)=\left\{\begin{array}{c}\frac{2}{b}(x-a) e^{-(x-a)^{2} / b} \\
0\end{array}\right.$ & $\begin{array}{l}z \geq a \\
z<a\end{array}$ & $\begin{array}{c}(0.02,0.3) \\
(0.06,0.2) \\
(0,1) \\
(0.1,0.4) \\
(0.2,0.3) \\
(0.3,0.1)\end{array}$ & $\begin{array}{c}E X=a+\sqrt{\pi b / 4} \\
D X=\frac{b(4-\pi)}{4}\end{array}$ \\
\hline
\end{tabular}

Fig. 4 shows the degraded images contaminated by gradually severer Gaussian noises of the Digit 3. It can be intuitively seen from the figures that with the increase of the parameter values, the images are gradually blurring off from left to right. The last image is not recognizable even by naked eyes. Under different levels of interference, the computed sensitivity of each layer in a CNN is 
compared with the simulating output variation in the following experiments to test the feasibility of the algorithm at varying levels of noises.
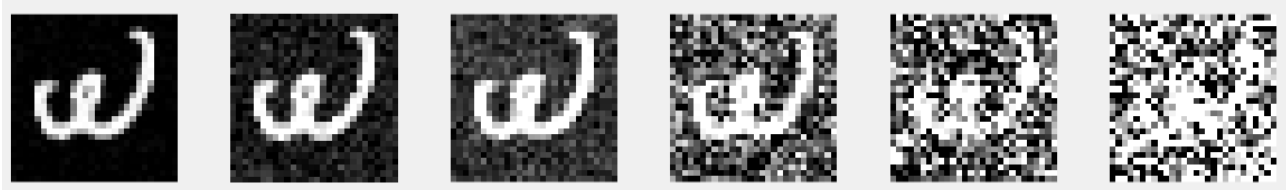

Fig. 4 Degraded images contaminated by different levels of noises in Gaussian distribution

Fig. 5 and Fig. 6 show the Chi-square distances between TS and SS of output feature maps at convolutional layers C1, C2, and pooling layers P1, P2. Distributions of values in Fig. 6 show that the Chi-square distances are always very close to zero. The last set of parameters perturbation $(0.5$, 0.8 ) causes the largest deviation on Map1, but it still does not exceed 0.2. All the others are in the order of $10^{-3}$ or $10^{-2}$, very close to zero. The results show that the algorithm CNN_SEN_INP is effective in computing the sensitivity under the influence of Gaussian noise at both convolutional and pooling layers.
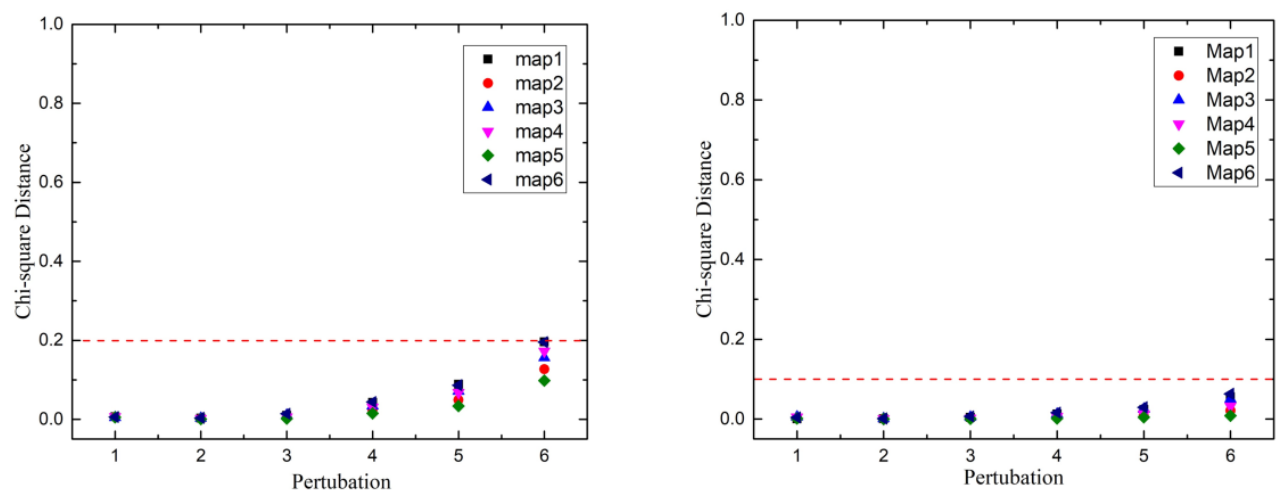

Fig. 5 The Chi-square distances between $T S$ and $S S$ of Map1-Map6 in C1(Left) and P1(Right) layer with Gaussian noises at six different intensity levels
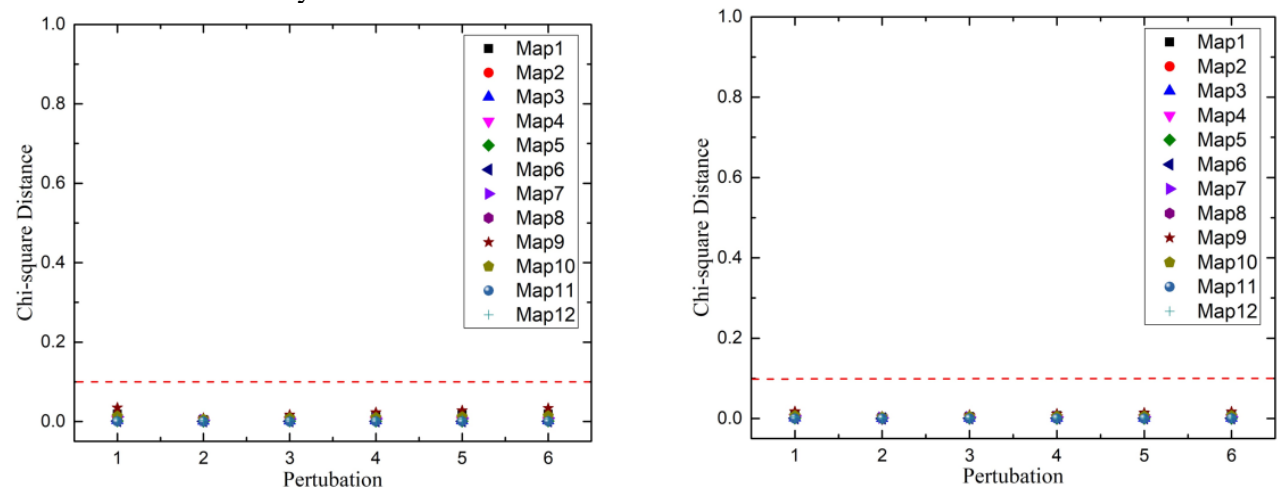

Fig. 6 The Chi-square distances between TS and SS of Map1-Map12 in C2(Left) and P2(Right) layer with Gaussian noises at six different intensity levels

Table 2 gives the Chi-square distances between $T S$ and $S S$ under 6 different levels of the Gaussian noises at the output layer. It is easy to see that all the results are close to zero. It shows that the sensitivity of the entire network computed by the proposed algorithm is almost the same as that of the actual output variation, which indicates the output variation of the network can be estimated accurately.

Table 2 The Chi-square distances between $T S$ and $S S$ at the output layer $(\mathrm{O})$ perturbed by additive Gaussian noises

\begin{tabular}{lllllll}
\hline \hline$(a, b)$ & $(0,0.02)$ & $(0.1,0.08)$ & $(0.2,0.1)$ & $(0.3,0.3)$ & $(0.4,0.5)$ & $(0.5,0.8)$ \\
\hline Distance & 0.00067717 & 0.0026 & 0.0013 & 0.0010 & 0.000073 & 0.000051 \\
\hline \hline
\end{tabular}

In the same way, Uniform, Salt \& Pepper and Rayleigh noises are added to the input samples. Fig. 7- Fig. 9 show the degraded images of Digit 3 contaminated by three types of noises at different 
levels. The corresponding perturbation parameters are set as listed in Table 1. According to the parameters, the initial distribution values for the algorithm CNN_SEN_INP are determined, and then $T S$ can be computed layer by layer.
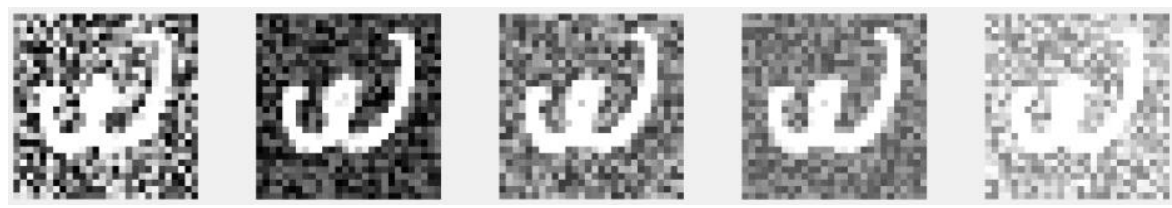

Fig. 7 Degraded images contaminated by different levels of noise with a Uniform distribution
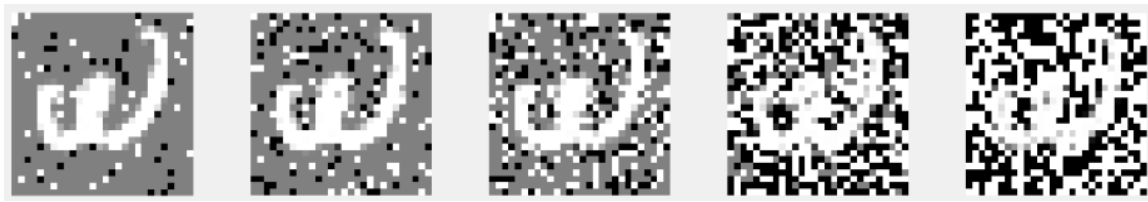

Fig. 8 Degraded images contaminated by different levels of noise with a Salt \& Pepper distribution
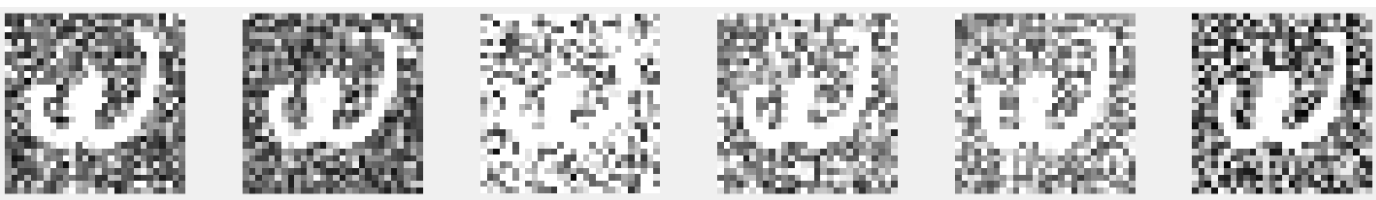

Fig. 9 Degraded images contaminated by different levels of noise with a Rayleigh distribution

Fig. 10 shows the Chi-square distances at layer $\mathrm{C} 1$ and $\mathrm{P} 1$ under five levels of Uniform noises. Almost all the values are close to 0 , and the maximum value is no more than 0.2. Similarly, the Chisquare distances of the twelve feature maps at layer $\mathrm{C} 2$ and $\mathrm{P} 2$ do not exceed 0.1 as shown in Fig. 11. The results show that the sensitivity of each layer computed by the algorithm CNN_SEN_INP can effectively represent the output variation of each layer under various levels of Uniform noises.
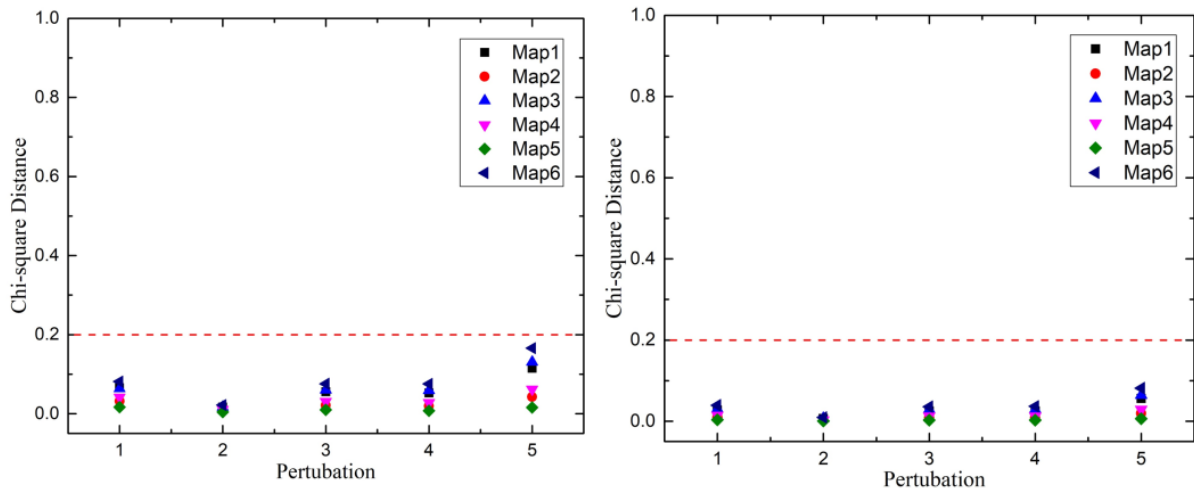

Fig. 10 The Chi-square distances between $T S$ and SS of Map1-Map6 at C1(Left) and P1(Right) layer with Uniform noises at five different levels
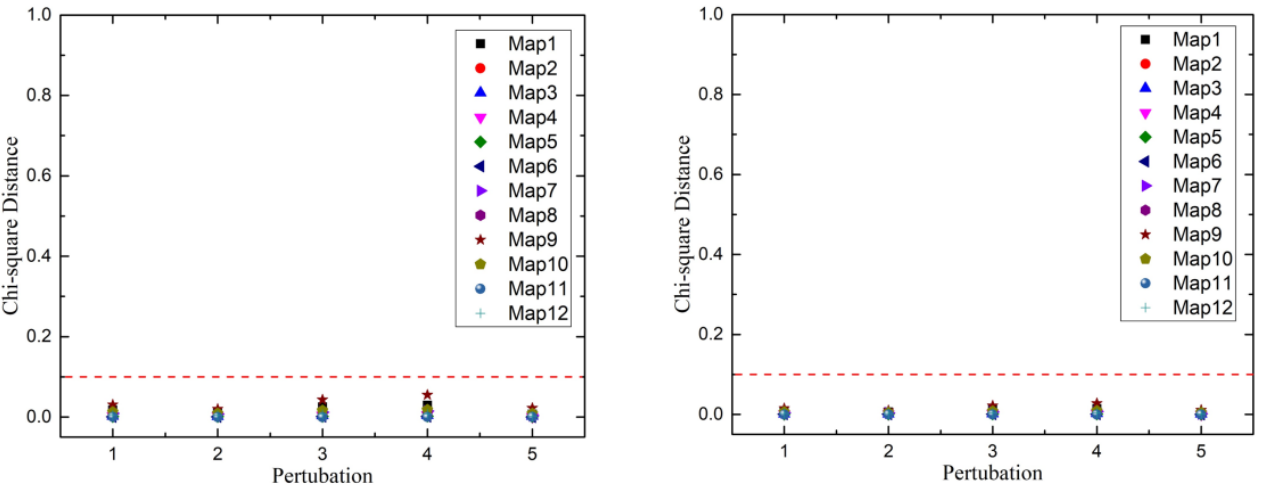

Fig. 11 The Chi-square distances between TS and SS of Map1-Map12 at C2(Left) and P2(Right) layer with Uniform noises at five different levels 
Table 3 The Chi-square distances between theoretical sensitivity (TS) and observed sensitivity (SS) at the output layer $(\mathrm{O})$ perturbed by Uniformly distributed noises

\begin{tabular}{cccccc}
\hline \hline$(a, b)$ & $(0,1)$ & $(0,0.5)$ & $(0.2,0.8)$ & $(0.3,0.7)$ & $(0.5,1)$ \\
\hline Distance & 0.000628 & 0.0011 & 0.00043 & 0.00034 & 0.00093 \\
\hline \hline
\end{tabular}

Table 3 lists the Chi-square distances between the sensitivity of the entire network and the output variation of CNN under five different levels of perturbation in Uniform distribution. We can see that the distance always keeps the same and even a significant increase of noise does not change much of it. Almost All those distance values are between $10^{-3}$ and $10^{-2}$, which demonstrates the feasibility of the proposed algorithm for the entire network against different levels of Uniform noise.
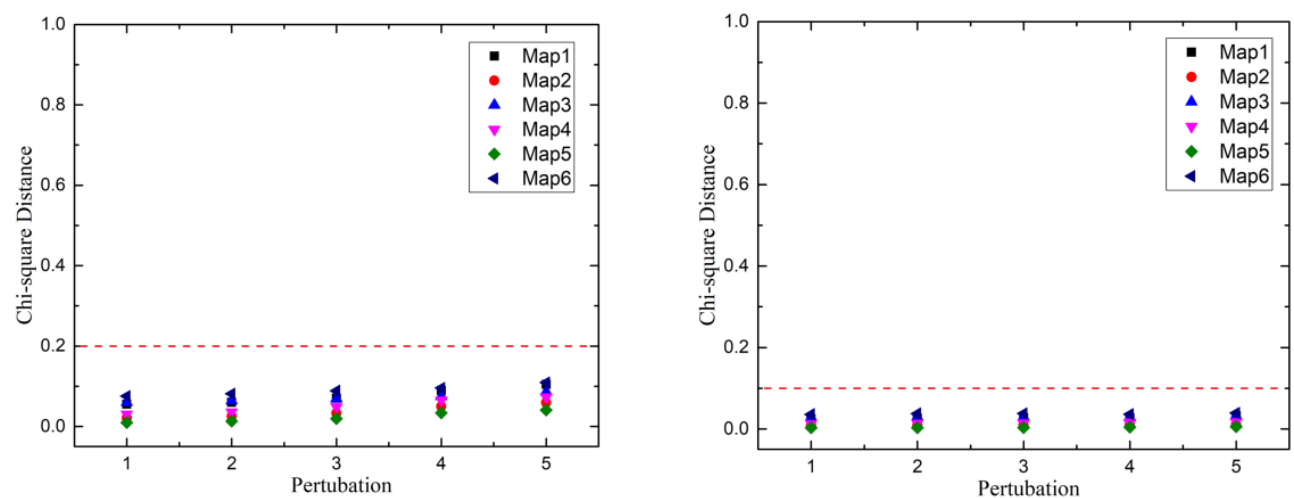

Fig. 12 The Chi-square distances between TS and SS of Map1-Map6 in C1(Left) and P1(Right) layer with Salt \& Pepper noises at five different levels
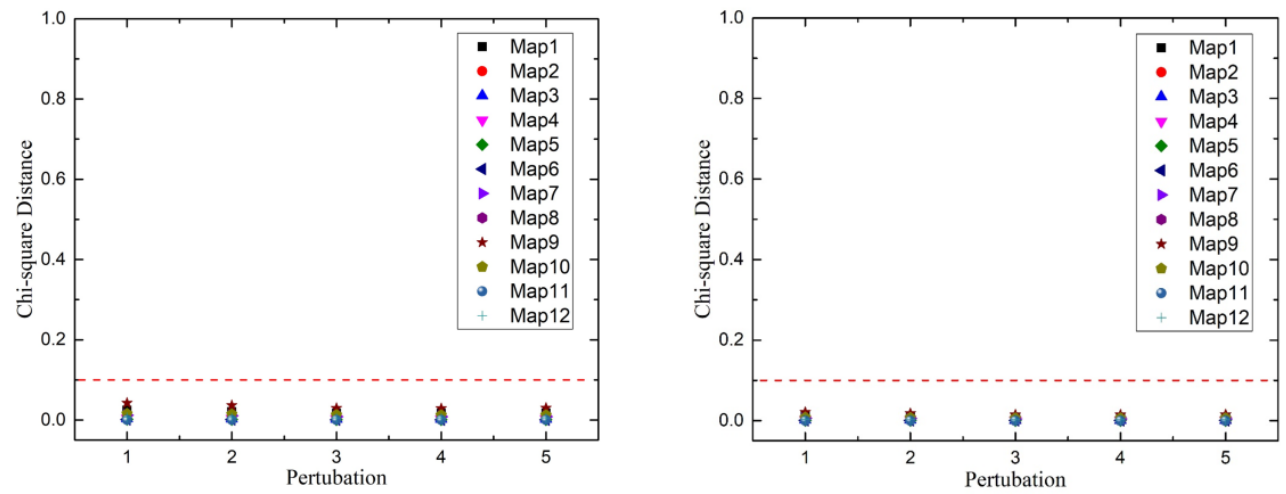

Fig. 13 Chi-square distances between $T S$ and $S S$ of Map1-Map12 in C2(Left) and P2(Right) layer with Salt \& Pepper noises at five different levels

Table 4 The Chi-square distances between theoretical sensitivity $(T S)$ and observed sensitivity $(S S)$ in the output layer (O) perturbed by Salt \& Pepper noises

\begin{tabular}{lccccc}
\hline \hline $\boldsymbol{a}=\boldsymbol{b}$ & 0.05 & 0.1 & 0.2 & 0.4 & 0.5 \\
\hline Distance & 0.00044 & 0.00051 & 0.00064 & 0.00069 & 0.00065 \\
\hline \hline
\end{tabular}

The Chi-square distances between the maps under the perturbations of Salt \& Pepper and Rayleigh noise are shown in Fig. 12-Fig. 15. With the Rayleigh noise, the calculated sensitivity of the first convolution layer $\mathrm{C} 1$ and the first pooling layer P1 differs greatly from the observed output variation, which is quite different from the situation of the Rayleigh noise, but the maximum value is no more than 0.25 . On the 12 feature maps at layers $\mathrm{C} 2$ and $\mathrm{P} 2$, the values are very close to 0 . Table 4 and Table 5 list the Chi-square distances between the computed sensitivity and the observed output variations of the entire network, perturbed by Salt \& Pepper and Rayleigh noises, respectively. Under these two different noise perturbations, the distances for the whole network are kept in the order of $10^{-4}$, which demonstrates the feasibility of the algorithm CNN_SEN_INP with both Salt \& Pepper and Rayleigh noise. 

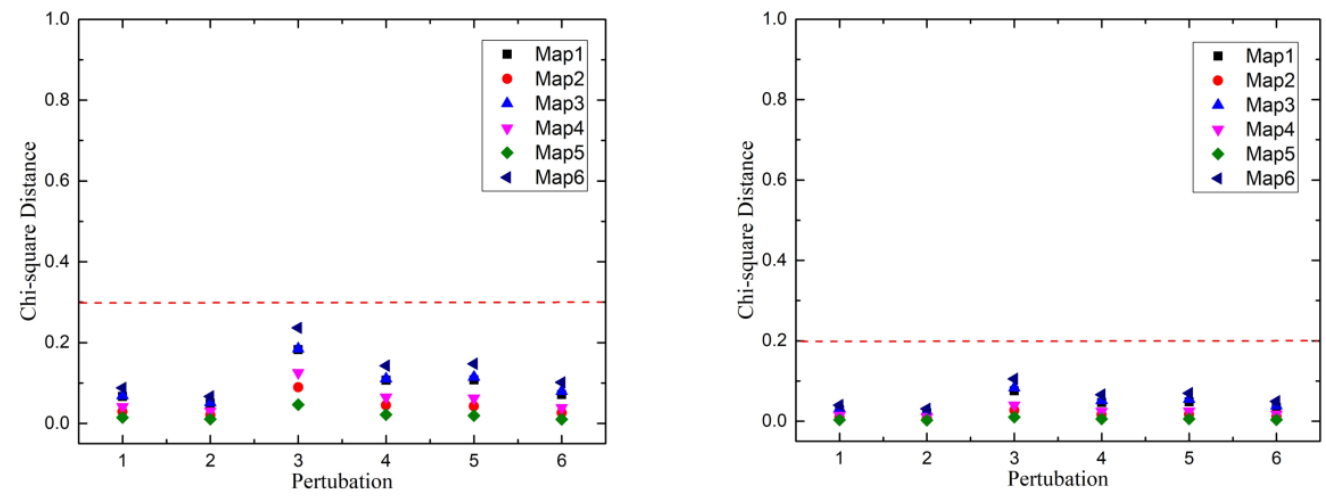

Fig. 14 The Chi-square distances between TS and SS of Map1-Map6 on C1(Left) and P1(Right) layer with Rayleigh noises at six different levels
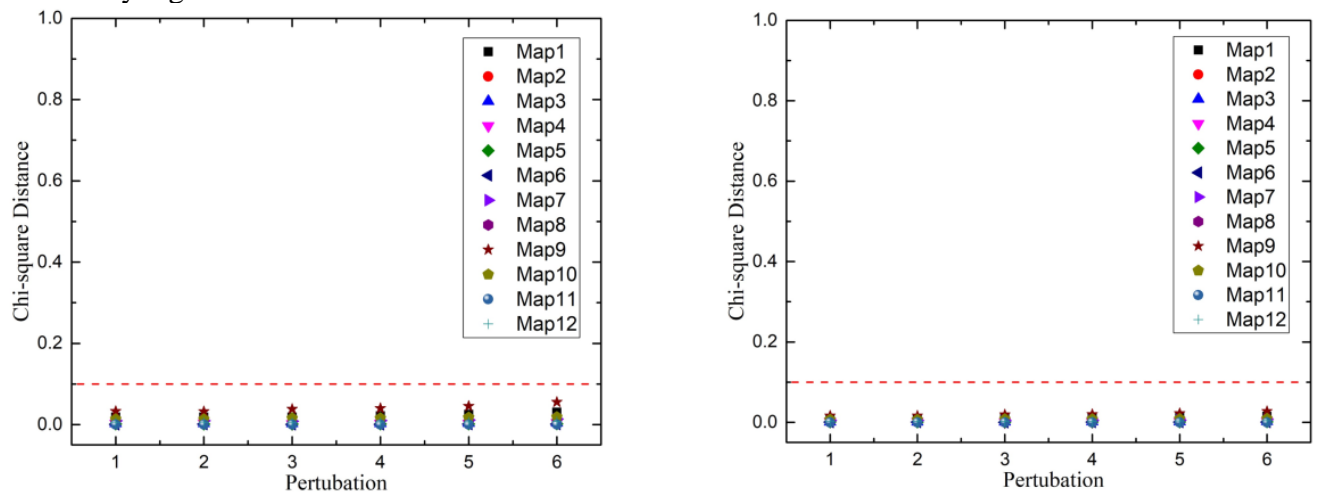

Fig. 15 The Chi-square distances between $T S$ and SS of Map1-Map12 on C2(Left) and P2(Right) layer with Rayleigh noise at six different levels

Table 5 The Chi-square distances between theoretical sensitivity $(T S)$ and observed sensitivity $(S S)$ on the output layer $(\mathrm{O})$ perturbed by the Rayleigh noises

\begin{tabular}{lllllll}
\hline \hline$(a, b)$ & $(0.02,0.3)$ & $(0.06,0.2)$ & $(0,1)$ & $(0.1,0.4)$ & $(0.2,0.3)$ & $(0.3,0.1)$ \\
\hline Distance & 0.00058 & 0.00061 & 0.00033 & 0.00039 & 0.00033 & 0.00031 \\
\hline \hline
\end{tabular}

In summary, our verification experiments demonstrate the consistency of the computed sensitivity by the iterative algorithm CNN_SEN_INP proposed in this paper with the observed output variations on both individual layers and the entire CNN under four types of common noises at varying intensity levels. These results confirm what we intuitively expected that the sensitivity can reflect the output variation of a CNN to input perturbations. Thus our approach is a good one to reflect the variation of CNN due to input perturbations numerically and be applicable to assess the robustness of CNNs with additive input noise.

\subsection{Experimental results and applicability discussion}

By the above simulation experiments, we verify the reasonability of the definition of CNN's sensitivity and the feasibility of the proposed algorithm CNN_SEN_INP working with input images under various levels and types of noise on the trained CNN with fixed parameters and structure. From a global point of view, the sensitivity of a CNN, that is, the sensitivity of its output layer, reflects the CNN's output variation due to its first layer's input perturbation. Fig. 16 shows TS and $S S$ values of the 10 neurons at the output layer. It can be seen that the $T S$ value is almost in the same order as that of SS. Furthermore, among all the output neurons, the $S S$ values (shown in red solid line) of neurons 1, 4, 6 and 9 are greater than that of other neurons. Similarly, the TS values computed by CNN_SEN_INP also shows the same pattern. The results are consistent with what we intuitively expect. A greater TS value of a neuron corresponds to a larger variation, which indicates that the neuron is more sensitive to input perturbations. By taking the maximum or averaging all output neurons' sensitivity inside a network, we can quantify the sensitivity of the entire network. 
Thus $T S$ can be used to measure the sensitivity of the whole CNN to input perturbation numerically, and provide a measurement for judging sensitive and non-sensitive neurons in a given CNN.

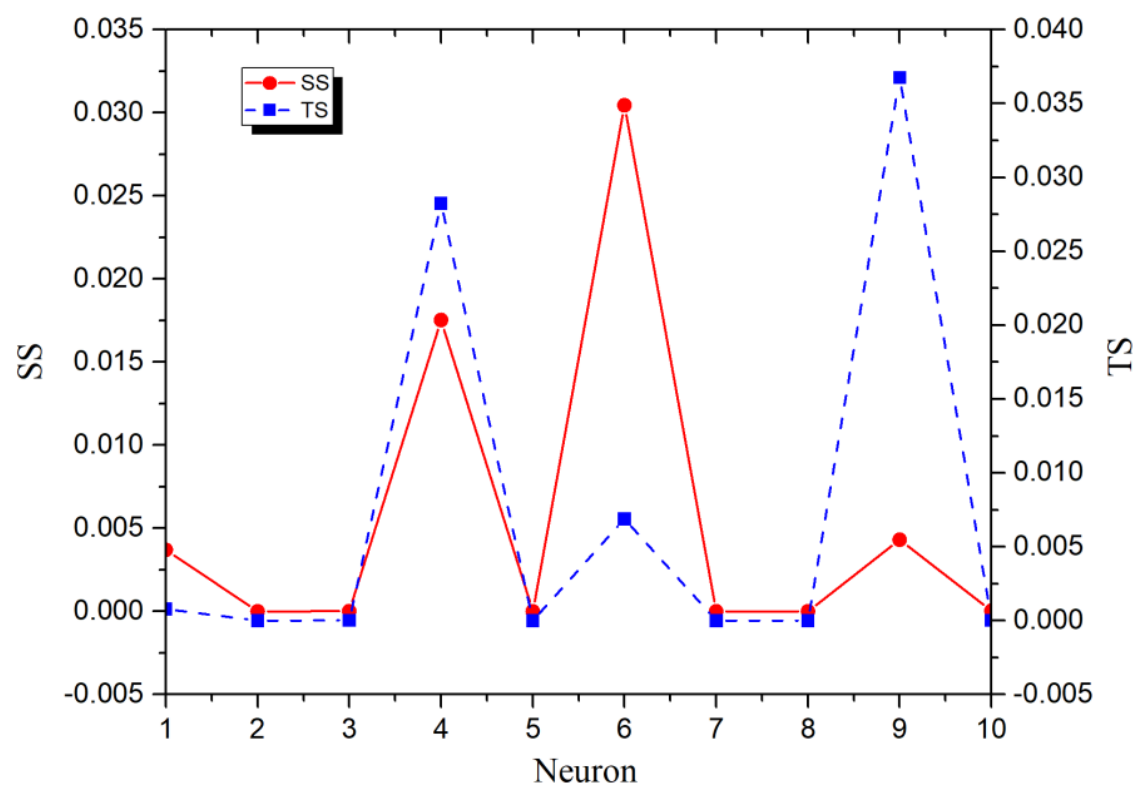

Fig.16 TS and SS of neurons in output layer of CNN-1

To test the effect of network depth on sensitivity, the algorithm is run on a group of CNNs with different layers, under which the same level of Gaussian noise perturbation applies. On the simplest architecture of C1(6,5,5)-P1(2,2)-F(192)-O(10) with an accuracy of $90.2 \%$, both $T S$ and $S S$ values of all the neurons at the output layer are very close to 0 . The maximal $T S$ is $6.0626 \mathrm{e}-15$ and the maximal $S S$ is $3.4062 \mathrm{e}-14$. It shows that the output is very much immutable to input noise and our algorithm works for networks with a small number of layers. Moreover, we also run our algorithm on a model with deeper layers as $\mathrm{C} 1(6,5,5)-\mathrm{P} 1(2,2)-\mathrm{C} 2(12,5,5)-\mathrm{P} 2(2,2)-\mathrm{C} 3(12,5,5)-\mathrm{F}(192)-\mathrm{O}(10)$, denoted as $\mathrm{CNN}-2$, similar to the classic architecture of LeNet-5. For the convenience of the network to deal with input, the images in the MNIST dataset are resized from $28 \times 28$ to $32 \times 32$. After training, the network's accuracy reaches $93.83 \%$ and its test accuracy is $93.45 \%$. Fig. 17 shows TS and $S S$ values of all the neurons at the output layer, with added Gaussian noise perturbation of the same level. It can be seen that the computed value $T S$ is very close to the observed value $S S$ and the two lines keep the similar pattern. A peak appears at neuron 4 for both. In the same way, we also experiment on a $\mathrm{CNN}$ with more layers than $\mathrm{CNN}-2 \mathrm{C} 1(6,5,5)-\mathrm{P} 1(2,2)-\mathrm{C} 2(6,5,5)-\mathrm{P} 2(2,2)-$ $\mathrm{C} 3(12,5,5)-\mathrm{P} 3(2,2)-\mathrm{C} 4(12,5,5)-\mathrm{F}(192)-\mathrm{O}(10)$, denoted as CNN-3. This time, the images in MNIST are resized to $92 \times 92$, the training accuracy is $95.35 \%$ and test accuracy is $95.21 \%$. The results show a similar tendency as the above two networks and the maximal $T S$ value is 0.1557 . It demonstrates that the proposed algorithm CNN_SEN_INP is also fit for deeper CNNs. Theoretically, the algorithm can deal with CNNs with any depth of layers. But, due to the limitation of computer hardware, further experiments on deeper $\mathrm{CNN}$ than $\mathrm{CNN}-3$ have not been conducted. Besides, the proposed algorithm is based on the basic convolutional neural network structure, without considering any variation of CNNs, e.g., with dropout and/or kernel regularization. In summary, the algorithm CNN_SEN_INP can compute not only the sensitivity of a network or a layer, but also that of a neuron, and it can be used as an alternative to measure the variation of each neuron's output at each layer due to outer input perturbations. 


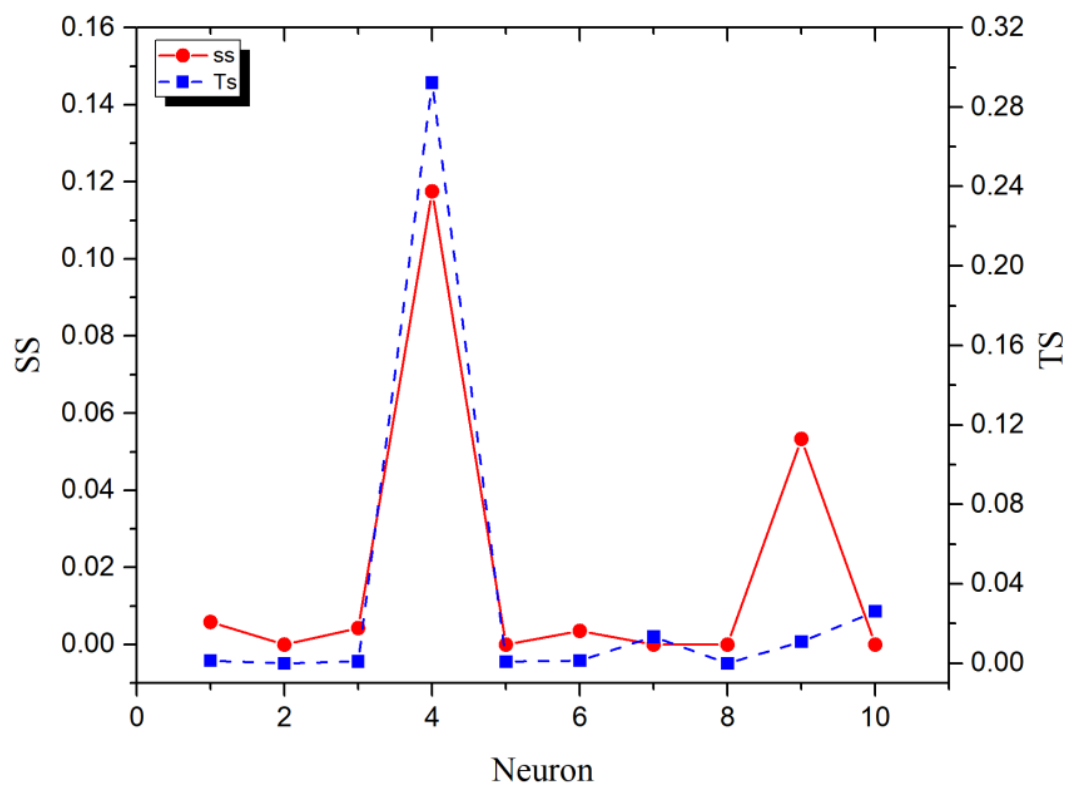

Fig. $17 T S$ and $S S$ values of neurons at output layer of $\mathrm{CNN}-2$

As for the practical applications, the computed sensitivity of the CNN model can be taken as a relative measure to tackle some important issues, for example robust model selection. Some experiments on $\mathrm{CNN}-1, \mathrm{CNN}-2$ and $\mathrm{CNN}-3$ have been conducted and the results show which model is not affected very much by noise, i.e., very robust against input noise. Random noises of Gaussian, Uniform and Rayleigh are added to the test images in the MNIST dataset. Fig. 18-20 show the variation rates of the three networks under three types of noise at different levels. To measure the variation after adding noise, the average variation of output (OVar) relative to the initial output (without adding any noise) is calculated as follow:

$$
O \operatorname{Var}=\left(\sum\left|O^{\prime}-O\right|\right) / n
$$

in which $O^{\prime}$ is the output of the network after adding random noises to input, while $O$ is the output of the network without adding any noise to input, and $n$ is the times of adding noises. Table 6 lists the average OVar of CNN-1, CNN-2 and CNN-3 under the Gaussian, Uniform and Rayleigh noise. It can be seen that the average $O$ Var of $\mathrm{CNN}-3$ is the smallest, and so the effect of input perturbation on CNN-3's output is the least. Under the same types and levels of noises, we also calculate the sensitivity of the three networks. Table 7 gives the TS values of CNN-1, CNN-2 and CNN-3. It shows that the CNN-3 gets the least, lower than the other two networks do. Thus, CNN-3 can be taken as the most robust model under the Gaussian, Uniform and Rayleigh noises. The consistency of the theoretical results with the experimental results demonstrates that $T S$ can be used for measuring the variation of output due to input perturbations. In addition, different from sensitivity analysis of CNNs by extensive experiments in [50], this paper gives a numerical value for quantitively measuring the sensitivity of CNNs, by which the performance of the network can be objectively and distinctly assessed. Moreover, sensitivity of each neuron in each trainable network layer can also be computed in a statistical way by the proposed algorithm CNN_SEN_INP, which is quite different from sensitivity analysis of the entire model from input to output in a geometric way, e.g. in [52]. 


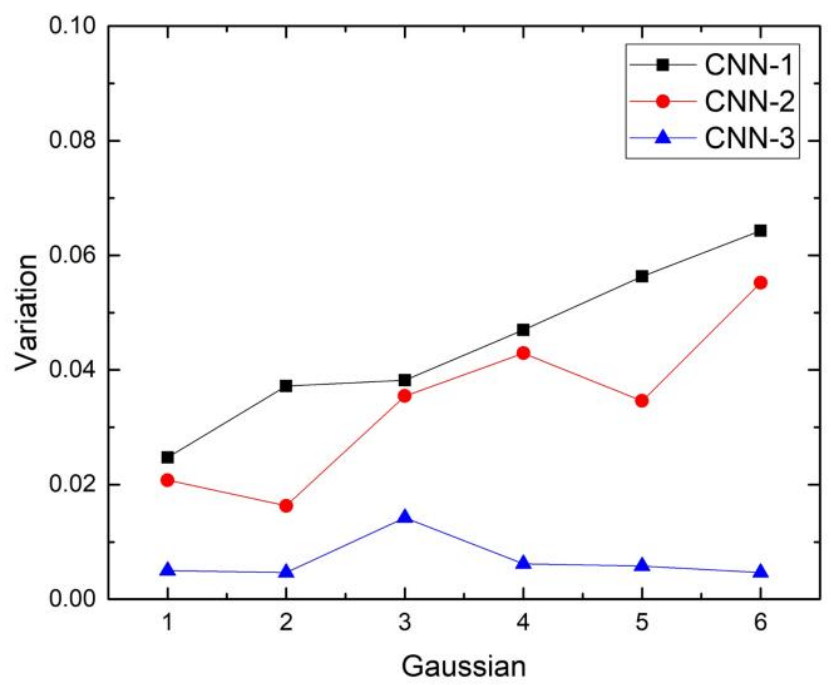

Fig. 18 Variation rates of $\mathrm{CNN}-1, \mathrm{CNN}-2$ and $\mathrm{CNN}-3$ caused by Gaussian noises

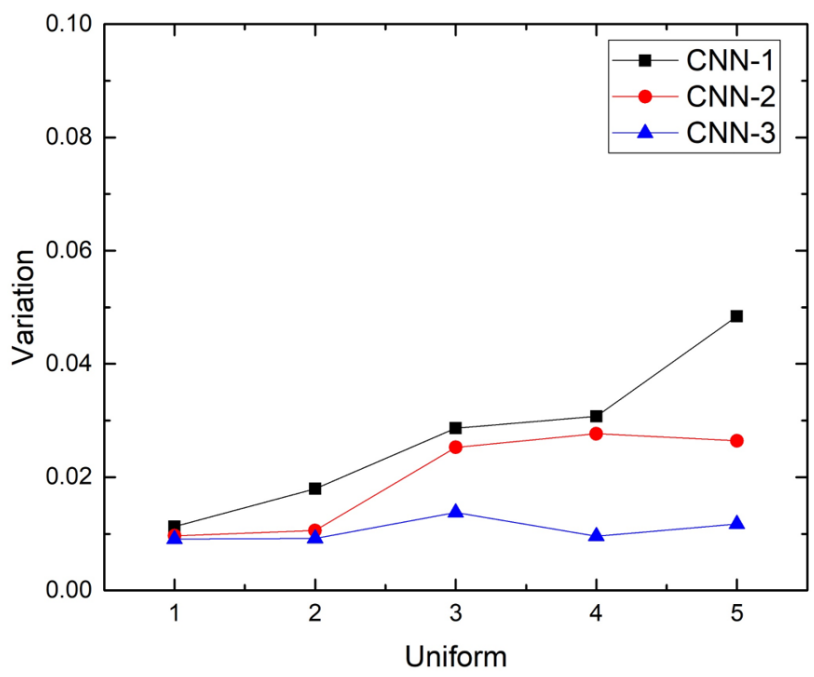

Fig. 19 Variation rates of $\mathrm{CNN}-1, \mathrm{CNN}-2$ and $\mathrm{CNN}-3$ caused by Uniform noises

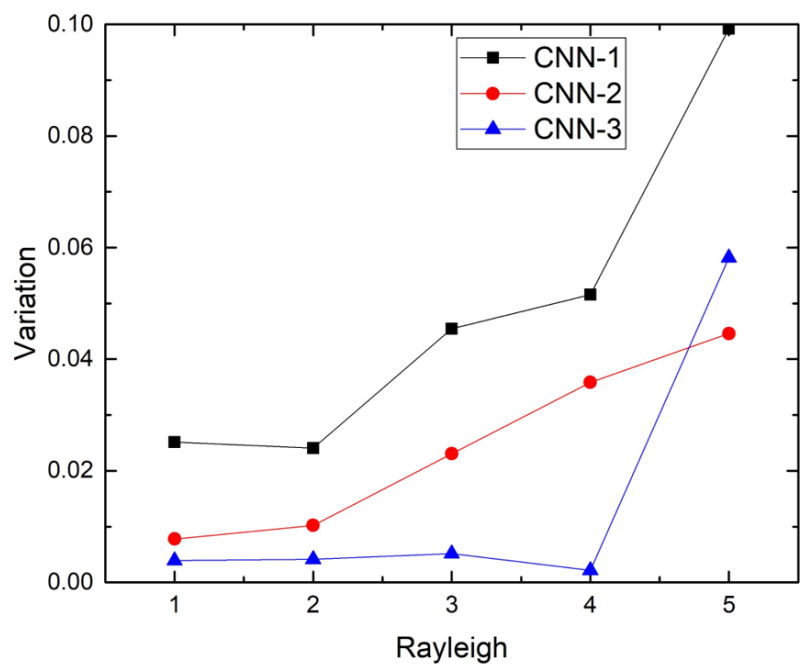

Fig. 20 Variation rates of $\mathrm{CNN}-1, \mathrm{CNN}-2$ and $\mathrm{CNN}-3$ caused by Rayleigh noises 
Table 6 OVar of CNN-1, CNN-2 and CNN-3 under Gaussian, Uniform and Rayleigh noises

\begin{tabular}{ccccc}
\hline \hline OVar & Gaussian & Uniform & Rayleigh & Average \\
\hline CNN-1 & 0.0446 & 0.0274 & 0.0491 & 0.0404 \\
\hline CNN-2 & 0.0342 & 0.0199 & 0.0243 & 0.0261 \\
\hline CNN-3 & 0.0067 & 0.0107 & 0.0147 & 0.0107 \\
\hline \hline
\end{tabular}

Table 7 TS values of CNN-1, CNN-2 and CNN-3 under Gaussian, Uniform and Rayleigh noises

\begin{tabular}{ccccc}
\hline \hline$T S$ & Gaussian & Uniform & Rayleigh & Average \\
\hline CNN-1 & 0.0271 & 0.0397 & 0.0393 & 0.0354 \\
\hline CNN-2 & 0.0230 & 0.0364 & 0.0368 & 0.0321 \\
\hline CNN-3 & 0.0172 & 0.0354 & 0.0247 & 0.0258 \\
\hline \hline
\end{tabular}

\section{Conclusion}

This paper focuses on building a criterion for measuring the sensitivity of CNNs' output to input perturbations and presents an approach for approximately quantifying the sensitivity. The sensitivity is defined in a bottom-up way from neuron to layer and finally to the entire network. Based on the definition, an algorithm called CNN_SEN_INP is designed for computing the value of the sensitivity. For some typical CNNs, the reasonability of the definition and the feasibility of the computation are experimentally verified. The experimental results show that the computed sensitivity (TS) agrees with the simulated sensitivity (SS) of every feature map and the final output layer, regardless of the setting on maps, layers and networks. Tests on some CNN models with deeper layers also demonstrate that CNN_SEN_INP works well for deeper networks under some basic assumptions. Besides, some tests on CNN models also show that sensitivity, as a relative criterion, can be applicable for robust model selection. In the future, the application of the sensitivity to prune the $\mathrm{CNN}$ with smaller architecture may be a worthy research direction.

\section{Acknowledgments}

This work is supported by the Fundamental Research Funds for the Central Universities under Grant No. 2016B44414 and 2018B678X14, Postgraduate Research \& Practice Innovation Program of Jiangsu Province of China under Grant No. KYCX18_0553, and the Science and Technology Project of Huai'an City under Grant No. HAG201602 and HAS201607.

\section{Reference}

[1] Krizhevsky A, Sutskever I, Hinton GE (2012) Imagenet classification with deep convolutional neural networks. Advances in neural information processing systems, pp 1097-1105

[2] Simon M, Rodner E (2015) Neural activation constellations: Unsupervised part model discovery with convolutional networks. In: Proceedings of the IEEE International Conference on Computer Vision:1143-1151

[3] Simonyan K, Zisserman A (2014) Very deep convolutional networks for large-scale image recognition. ArXiv preprint. ArXiv:1409.1556

[4] Karpathy A et al (2014) Large-scale video classification with convolutional neural networks. In: Proceedings of the IEEE Conference on Computer Vision and Pattern Recognition:1725-1732

[5] Girshick R et al (2014) Rich feature hierarchies for accurate object detection and semantic segmentation. In: Proceedings of the IEEE conference on computer vision and pattern recognition: 580-587

[6] Kalchbrenner N, Grefenstette E, Blunsom P (2014) A convolutional neural network for modeling sentences. arXiv preprint. arXiv: 1404.2188 
[7] Zhang Y, Wallace B (2015) A sensitivity analysis of (and practitioners' guide to) convolutional neural networks for sentence classification. arXiv preprint. arXiv: 1510.03820

[8] Brust CA et al (2015) Convolutional patch networks with spatial prior for road detection and urban scene understanding. arXiv preprint. arXiv:1502.06344

[9] Hariharan B et al (2015) Hypercolumns for object segmentation and fine-grained localization. In: Proceedings of the IEEE conference on computer vision and pattern recognition: 447-456

[10] Fukushima K, Miyake S (1982) Neocognitron: A self-organizing neural network model for a mechanism of visual pattern recognition, Competition and cooperation in neural nets. Springer, Berlin, Heidelberg: 267-285

[11] Zeiler MD, Fergus R (2014) Visualizing and understanding convolutional networks. In: European conference on computer vision: 818-833

[12] Rodner E et al (2016) Fine-grained recognition in the noisy wild: Sensitivity analysis of convolutional neural networks approaches. arXiv preprint. arXiv:1610.06756

[13] Kwon S et al (2016). Measuring error-tolerance in SRAM architecture on hardware accelerated neural network. In: 2016 IEEE International Conference on Consumer Electronics- Asia:1-4

[14] Szegedy C et al (2013) Intriguing properties of neural networks. arXiv preprint. arXiv: 1312.6199

[15] Fawzi A, Fawzi O, Frossard P (2018) Analysis of classifiers' robustness to adversarial perturbations. Machine Learning 107(3):481-508

[16] Fawzi A et al (2016) Robustness of classifiers: from adversarial to random noise. In: Advances in Neural Information Processing Systems:1632-1640

[17] Moosavi D, Seyed M (2017) Universal adversarial perturbations. In: Proceedings of the IEEE conference on computer vision and pattern recognition: 86-94

[18] Sharif M et al (2016) Accessorize to a crime: Real and stealthy attacks on state-of-the-art face recognition. In: Proceedings of the 2016 ACM SIGSAC Conference on Computer and Communications Security:1528-1540

[19] Akhtar N, Mian A (2018) Threat of adversarial attacks on deep learning in computer vision: A survey. arXiv preprint arXiv:1801.00553

[20] Novak R el al (2018) Sensitivity and generalization in neural networks: an empirical study. In International Conference on Learning Representations. arXiv:1802.08760.

[21] Moosavi D el al (2016) Deepfool: a simple and accurate method to fool deep neural networks. In Proceedings of the IEEE Conference on Computer Vision and Pattern Recognition: 2574-2582

[22] Hein M, Andriushchenko M (2017) Formal guarantees on the robustness of a classifier against adversarial manipulation. In Advances in Neural Information Processing Systems: 2266-2276.

[23]Zhang, J., Huang, C (2020) Dynamics analysis on a class of delayed neural networks involving inertial terms. Adv Differ Equ 2020, 120. https://doi.org/10.1186/s13662-020-02566-4

[24]Yang, X et al (2019) Dynamic Properties of Foreign Exchange Complex Network. Mathematics, 7,832 .

[25]Huang C , Tan Y(2020) Global behavior of a reaction-diffusion model with time delay and Dirichlet condition[J]. Journal of Differential Equations, 271:186-215.

[26] Fawzi A et al (2017) The robustness of deep networks: A geometrical perspective. IEEE Signal Processing Magazine 34(6):50-62

[27] Saltelli A (2002) Sensitivity analysis for importance assessment. Risk Anal 22(3):579-590 
[28] Saltelli A et al (2019) Why so Many Published Sensitivity Analyses Are False: A Systematic Review of Sensitivity Analysis Practices. Environmental Modelling and Software 114: 29-39

[29] Saltelli A et al (2008) Global sensitivity analysis: the primer. Wiley, Chichester, England

[30] Veiga D et al (2015) Global sensitivity analysis with dependence measures. Journal of Statistical Computation \& Simulation 85 (7): 1283-1305

[31] Stevenson M, Winter R, Widrow B (1990) Sensitivity of feedforward neural networks to weight errors. IEEE Transactions on Neural Networks 1(1):71-80

[32] Piche SW et al (1995) The selection of weight accuracies for Madalines. IEEE Transactions on Neural Networks 6(2): 432-445

[33] Zeng X, Wang Y, Zhang K (2006) Computation of Adalines' sensitivity to weight perturbation. IEEE transactions on neural networks 17(2): 515-519

[34] Wang Y et al (2006) Computation of Madalines' sensitivity to input and weight perturbations. Neural computation 18(11):2854-2877

[35] Choi JY, Choi CH (1992) Sensitivity analysis of multilayer perceptron with differentiable activation functions. IEEE Transactions on Neural Networks 3(1): 101-107

[36] Fu L, Chen T (1993) Sensitivity analysis for input vector in multilayer feedforward neural networks. In: IEEE International Conference on Neural Networks:215-218

[37] Yeung D, Sun X (2002) Using function approximation to analyze the sensitivity of MLP with antisymmetric squashing activation function. IEEE Transactions on Neural Networks 13(1):34-44

[38] Yang S, Ho C, Siu S (2007) Sensitivity analysis of the split-complex valued multilayer perceptron due to the errors of the iid inputs and weights. IEEE transactions on neural networks 18(5): 1280-1293

[39] Zeng X, Yeung D (2001) Sensitivity analysis of multilayer perceptron to input and weight perturbations. IEEE Transactions on Neural Networks 12(6):1358-1366

[40] Zeng X, Yeung D (2003) A quantified sensitivity measure for multilayer perceptron to input perturbation. Neural Computation 15(1): 183-212

[41] Ng WWY et al (2002) Statistical output sensitivity to input and weight perturbations of radial basis function neural networks. In: IEEE International Conference on Systems, Man and Cybernetics2: 503-508

[42] Cheng A, Yeung D (1999) Sensitivity analysis of neocognitron. IEEE Transactions on Systems, Man, and Cybernetics, Part C (Applications and Reviews) 29(2):238-249

[43] Chen D et al (2020) Fixed time synchronization of delayed quaternion-valued memristor- based neural Networks. Advances in Difference Equations. 2020, 92. https://doi.org/10.1186/ s13662-020 $-02560-\mathrm{w}$

[44] Cao JD et al (2020) Zagreb Connection Indices of Molecular Graphs Based on Operations. Complexity, vol. 2020: 1-15

[45] Zhou Y et al (2020) Finite-Time Stochastic Synchronization of Dynamic Networks with Nonlinear Coupling Strength via Quantized Intermittent Control. Applied Mathematics and Computation, vol. 376:125157

[46] Yeung D et al (2010) Sensitivity Analysis for Neural Networks[M]. Springer Berlin Heidelberg [47] Wang W et al (2020) Bipartite Formation Problem of Second-Order Nonlinear Multi-Agent Systems with Hybrid Impulses. Applied Mathematics and Computation, vol. 370: 124926

[48] Huang C et al (2020) Asymptotic Behavior for a Class of Population Dynamics. Math 5(4):3378-3390 
[49] Kumari, Sudesh et al (2020) On the Construction, Properties and Hausdorff Dimension of Random Cantor One pth Set. Math 2020,5(4) :3138-3155

[50] Zhang Y, Wallace B (2017) Sensitivity Analysis of (and Practitioners' Guide to) Convolutional Neural Networks for Sentence Classification. In: international joint conference on natural language processing: 253-263

[51] Rawat W, Wang Z (2017) Deep convolutional neural networks for image classification: A comprehensive review. Neural computation 29(9): 2352-2449

[52] Shu H, Zhu H (2019) Sensitivity Analysis of Deep Neural Networks. In Proceedings of the AAAI Conference on Artificial Intelligence 33: 4943-4950 